\title{
Searching for massive clusters in weak lensing surveys
}

\author{
Takashi Hamana ${ }^{1,2}$, Masahiro Takada ${ }^{3}$ and Naoki Yoshida ${ }^{2,4}$ \\ ${ }^{1}$ Institut d'Astrophysique de Paris, 98bis Boulevard Arago, F 75014 Paris, France \\ ${ }^{2}$ National Astronomical Observatory of Japan, Mitaka, Tokyo 181-8588, Japan \\ ${ }^{3}$ Department of Physics and Astronomy, University of Pennsylvania, 209 S. 33rd Street, Philadelphia, PA 19104, USA \\ ${ }^{4}$ Harvard-Smithsonian Center for Astrophysics, 60 Garden Street, Cambridge, MA 02138, USA
}

Accepted 30 January 2004; Received 30 January 2004; in original form 21 October 2003

\begin{abstract}
We explore the ability of weak lensing surveys to locate massive clusters. We use both analytic models of dark matter halos and mock weak lensing surveys generated from a large cosmological $N$-body simulation. The analytic models describe average properties of weak lensing halos and predict the number counts, enabling us to compute an effective survey selection function. We argue that the detectability of massive halos depends not only on the halo mass but also strongly on redshift at which the halo is located. We test the model prediction for the peak number counts in weak lensing mass maps against the mock numerical data, and find that the noise due to intrinsic galaxy ellipticities causes a systematic effect which increases the peak counts. We develop a correction scheme for the systematic effect in an empirical manner, and show that, after the correction, the model prediction agrees well with the mock data. The mock data is also used to examine the completeness and efficiency of the weak lensing halo search with fully taking into account the noise and the projection effect by large-scale structures. We show that the detection threshold of $\mathrm{S} / \mathrm{N}=4 \sim 5$ gives an optimal balance between completeness and efficiency. Our results suggest that, for a weak lensing survey with a galaxy number density of $n_{g}=30 \mathrm{arcmin}^{-2}$ with a mean redshift $z=1$, the mean number of halos which are expected to cause lensing signals above $\mathrm{S} / \mathrm{N}=4$ is $N_{\text {halo }}(\mathrm{S} / \mathrm{N}>4)=37$ per $10 \mathrm{deg}^{2}$, whereas 23 of the halos are actually detected with $\mathrm{S} / \mathrm{N}>4$, giving the effective completeness as good as $63 \%$. On the other hand, the mean number of peaks in the same area is $N_{\text {peak }}=62$ for a detection threshold $\mathrm{S} / \mathrm{N}=4$. Among the 62 peaks, 23 are due to halos with the expected peak height $\mathrm{S} / \mathrm{N}>4,13$ are due to halos with $3<\mathrm{S} / \mathrm{N}<4$ and the remaining 26 peaks are either the false peaks due to the noise or halos with a lower expected peak height. Therefore the contamination rate is $42 \%$ (this could be an overestimation). Weak lensing surveys thus provide a reasonably efficient way to search for massive clusters.
\end{abstract}

Key words: cosmology: theory — gravitational lensing — galaxies: halos — dark matter — large-scale structure of universe

\section{INTRODUCTION}

Recently, it has become feasible to locate massive clusters directly as density enhancements using weak gravitational lensing. Miyazaki et al. (2002) indeed discovered many clusters in a weak lensing halo survey over a 2.1 square degree field, proving the ability of weak lensing to identify massive clusters (see also Wittman et al. 2001). Unlike conventional optical or X-ray selected cluster catalogs, weak lensing cluster catalogs are free from a bias toward the luminous objects because the cluster finding is not based on the flux enhancement but on the projected mass enhancement. This is a great advantage of gravitational lensing. However, lensing has its own disadvantages as every other cluster survey technique (optical, X-ray and Sunyaev-Zel'dovich survey) does; for example, the projection effect from unrelated structures in the same line-of-sight (Reblinsky \& Bartelmann 1999; Metzler, White \& Loken 2001).
Since clusters of galaxies are one of most important cosmological probes, it is of fundamental importance to construct large unbiased samples. Given advantages and disadvantages in each survey technique, it is desirable to combine, in a complementary manner, several techniques to construct an unbiased catalog. Such catalogs provide a valuable data set to investigate, for example, the physical state of the intra-cluster medium. To do this, it is important to correctly understand the selection function and completeness of each survey technique.

It has been often argued that weak lensing provides a truly mass-selected sample of clusters. This is not strictly true; weak lensing measures the two-dimensional tidal field which is a lineof-sight projection of the three-dimensional tidal field weighted by distance ratio $D_{l} D_{l s} / D_{s}$ (see $\S 2$ for definitions and details; see Mellier 1999; Bartelmann \& Schneider 2001 for reviews of weak lensing). In simpler terms, the amplitude of the lensing signal from 
a cluster is not solely determined by its mass, but depends also on the redshift and the shape of the gravitational potential due to the cluster. In addition, both foreground and background large-scale structures contribute to the lensing signal, and this projection effect usually adds a noise to the lensing signal from a halo. Further, since the weak lensing signal is measured from tiny coherent distortions in galaxy shapes, intrinsic ellipticities of galaxies introduce an irremovable noise. Thus, the detectability of weak lensing halo depends on all these factors.

The primary purpose of the present paper is to explore the ability of weak lensing surveys to locate massive clusters. We especially address the following three points; (1) to examine the selection function of weak lensing cluster surveys, (2) to develop a theoretical model that describes the weak lensing halo counts, and (3) to examine how the detailed structure of clusters, projection effect and noise affect weak lensing cluster surveys under a typical observational condition from a ground based $4-10 \mathrm{~m}$ class telescope. The last point is especially important to understand a bias that weak lensing halo catalogs may have. To address these things, we use both simple analytic descriptions of dark matter halos and mock numerical weak lensing survey data. The former offers a useful way to compute expected lensing properties of massive halos. We use them to compute the selection function. We also develop a theoretical model for the number counts of the weak lensing halos based on the analytic descriptions. On the other hand, the latter allows us to examine all the factors listed above in a direct manner. Mock numerical catalogs are generated using weak lensing raytracing simulations. We extensively use the mock data and the halo catalogs directly produced from the simulation outputs to examine completeness and efficiency of the weak lensing halo survey. The mock data is also used to test the model prediction of the weak lensing halo counts.

White, Van Waerbeke, \& Mackey (2002) and Padmanabhan, Seljak \& Pen (2003) addressed the ability of weak lensing surveys focusing on the projection effect. In those studies, the completeness of weak lensing surveys is defined as the fraction of detected halos with mass above a certain value relative to halos that lie in a given volume. This definition compromises two completely different elements, (i) the selection effect and (ii) the effects from individuality of the halo mass distribution, the projection and noise. For our purpose, these elements needs to be examined separately. We first compute the selection function using the analytical descriptions of dark matter halos which enables us to define "potentially detectable halos". Here, "potentially" refers to an ideal case in the absence of noise. Then, we shall adopt the different definition of the completeness that is the fraction of actually detected weak lensing halos relative to the potentially detectable halos.

Our results have important implications to real observations. When making an observational strategy, one would like to compute the expected number of detectable clusters using, for example, a simplified model, and also needs to estimate the expected completeness and false detection rate. Our theoretical model of the weak lensing halo counts may be useful for the former case, and our results from the mock data are directly applicable to derive the latter estimates. Also, from the observational point of view, we adopt a directly observable quantity, a peak height of weak lensing mass map divided by the noise root-mean-square (RMS), that is the signal-to-noise ratio, $\mathrm{S} / \mathrm{N}$, of the detection, as the fundamental estimate of the lensing signal.

The rest of this paper is organized as follows. In $\S 2$, we summarize lensing properties of massive halos assuming the universal density profile proposed by Navarro, Frenk \& White (Navarro,
Frenk \& White 1996; 1997). Using the expected properties, we compute the selection function of the weak lensing cluster survey. Then, we combine the detectability of weak lensing halos with the halo mass function based on the Press-Schechter approach (Press $\&$ Schechter 1974; we adopt the modified fitting function by Sheth \& Tormen 1999) to compute the weak lensing halo counts and the selection functions with respect to the halo mass and redshift. This simple analytical approach should offer a theoretical basis to explore properties of weak lensing halos. In $\S 3$, we describe our numerical experiment of weak lensing surveys and weak lensing ray-tracing technique. Statistical properties of halo catalogs are obtained and compared with the theoretical predictions derived in $\S 2$. Using the mock weak lensing survey data, in $\S 4$, we examine the number counts of peaks in the weak lensing convergence map, and the theoretical prediction developed in $\S 2$ is tested. In $\S 5$, we investigate correspondences between halos in the halo catalog and peaks identified in the lensing convergence map. In particular, we examine the completeness and efficiency of the weak lensing halo search paying special attention to the points (1-3) above. Summary and discussion are given in $\S 6$. In Appendix A, we present some lensing convergence maps in which a missing halo or a false peak exists for illustrative examples of irregular systems. In Appendix $\mathrm{B}$, we develop a correction scheme to the theoretical prediction of the weak lensing peak counts. In Appendix C, relations between the halo shape (and orientation) and the strength of lensing signal are examined.

Throughout this paper, we work with the standard flat $\Lambda \mathrm{CDM}$ cosmological model with the density parameter $\Omega_{\mathrm{m}}=0.3$, the cosmological constant $\Omega_{\Lambda}=0.7$, and Hubble constant $H_{0}=$ $100 h \mathrm{~km} \mathrm{~s}^{-1} \mathrm{Mpc}^{-1}$ with $h=0.7$. We adopt the fitting function of the CDM power spectrum by Bardeen et al. (1986) with the normalization of $\sigma_{8}=0.9$. We take the observational parameters of a typical weak lensing survey from a ground based $4-10 \mathrm{~m}$ telescope at the present. To be specific, we consider an imaging observation with a limiting magnitude fainter than $R=25.5$ mag in a sub-arcsecond seeing condition, which provides the galaxy number density $n_{g} \gtrsim 30 \operatorname{arcmin}^{-2}$ with the mean redshift $z \simeq 1$.

\section{PREDICTIONS FROM STANDARD MODELS}

\subsection{Lensing properties of an NFW halo}

The universal density profile of dark matter halos proposed by Navarro, Frenk \& White (1996; 1997; NFW hereafter) is given by

$\rho_{\mathrm{nfw}}(x)=\frac{\rho_{s}}{x(1+x)^{2}}, \quad x=\frac{r}{r_{s}}$,

where $r_{s}$ is the scale radius. It is convenient to introduce the concentration parameter $c_{\mathrm{nfw}}=r_{\mathrm{vir}} / r_{s}$ where $r_{\mathrm{vir}}$ is the virial radius. Bullock et al. (2001) found from $N$-body simulations that the concentration parameter is related to halo mass as

$c_{\mathrm{nfw}}(M, z)=\frac{c_{*}}{1+z}\left(\frac{M}{10^{14} h^{-1} M_{\odot}}\right)^{-0.13}$,

where we set $c_{*}=8$ for the $\Lambda \mathrm{CDM}$ model. It is important to notice that there is a relatively large scatter in this relation (Jing 2000). The mass enclosed within a sphere of radius $r_{\text {vir }}$ gives the virial mass by definition:

$M_{\mathrm{vir}}=\frac{4 \pi \rho_{s} r_{\mathrm{vir}}^{3}}{c_{\mathrm{nfw}}^{3}}\left[\log \left(1+c_{\mathrm{nfw}}\right)-\frac{c_{\mathrm{nfw}}}{1+c_{\mathrm{nfw}}}\right]$. 
The virial mass is also defined by the spherical top-hat collapse model as

$M_{\mathrm{vir}}=\frac{4 \pi}{3} \delta_{\mathrm{vir}}(z) \bar{\rho}_{0} r_{\mathrm{vir}}^{3}$,

where $\delta_{\text {vir }}$ is the threshold over-density for spherical collapse (see Nakamura \& Suto 1997 and Henry 2000 for useful fitting functions). From equations (3) and (4) one can express $\rho_{s}$ in terms of $\delta_{\mathrm{vir}}(z)$ and $c_{\mathrm{nfw}}$. Introducing $\delta_{s}=\rho_{s} / \bar{\rho}_{0}-1$, one finds,

$\delta_{s}=\frac{\delta_{\mathrm{vir}}}{3} \frac{c_{\mathrm{nfw}}^{3}}{\log \left(1+c_{\mathrm{nfw}}\right)-c_{\mathrm{nfw}} /\left(1+c_{\mathrm{nfw}}\right)}$.

Although the NFW profile formally extends to the infinity, an actual halo has a finite extent. We consider a truncated NFW halo, the density profile of which is truncated at the virial radius. The surface mass density of the truncated NFW halo is given by the lineof-sight integration of the density profile (Takada \& Jain 2003):

$\Sigma(y)=\int_{-\sqrt{c_{\mathrm{nfw}}^{2}-y^{2}}}^{\sqrt{c_{\mathrm{nfw}}^{2}-y^{2}}} d z \rho_{\mathrm{nfw}}(\boldsymbol{y}, z)=2 \rho_{s} r_{s} f(y), \quad y=\frac{r}{r_{s}},(6)$

with

$f(y)=\left\{\begin{array}{lr}-\frac{\sqrt{c_{\mathrm{nfw}}^{2}-y^{2}}}{\left(1-y^{2}\right)\left(1+c_{\mathrm{nfw}}\right)}+\frac{1}{\left(1-y^{2}\right)^{3 / 2}} \operatorname{arccosh} \frac{y^{2}+c_{\mathrm{nfw}}}{y\left(1+c_{\mathrm{nfw}}\right)} & \text { for } y<1 \\ \frac{\sqrt{c_{\mathrm{nfw}}^{2}-1}}{3\left(1+c_{\mathrm{nfw}}\right)}\left(1+\frac{1}{1+c_{\mathrm{nfw}}}\right), & \text { for } y=1, \\ -\frac{\sqrt{c_{\mathrm{nfw}}^{2}-y^{2}}}{\left(1-y^{2}\right)\left(1+c_{\mathrm{nfw}}\right)}-\frac{1}{\left(y^{2}-1\right)^{3 / 2}} & \arccos \frac{y^{2}+c_{\mathrm{nfw}}}{y\left(1+c_{\mathrm{nfw}}\right)} \\ 0, \quad \text { for } y>c . & \text { for } 1<y \leqslant c\end{array}\right.$

Note that the above expression differs from that by Bartelmann (1996) and Wright \& Brainerd (2000) which is for the nontruncated NFW halo and gives a larger surface mass density than ours. The lensing convergence profile is given by

$\kappa(y)=\frac{\Sigma(y)}{\Sigma_{c r}}=\kappa_{s} f(y)$

with the critical surface mass density

$\Sigma_{c r}=\frac{c^{2}}{4 \pi G} \frac{D_{s}}{D_{l} D_{l s}}$

and

$\kappa_{s}=3 \Omega_{\mathrm{m}} \delta_{s} r_{s}\left(\frac{H_{0}}{c}\right)^{2} \frac{\chi_{l}\left(\chi_{s}-\chi_{l}\right)}{a\left(z_{l}\right) \chi_{s}}$,

where $a$ is the scale factor normalized at the present time, $D$ denotes the angular diameter distance, $\chi$ denotes the comoving radial distance (which is equal to the comoving angular diameter distance for a flat universe), and the subscripts $l$ and $s$ stand for lens and source, respectively. It is worth noting that $\kappa$ scales with $c_{\mathrm{nfw}}$ approximately as $\kappa \propto c_{\mathrm{nfw}}$ for $1 \lesssim c_{\mathrm{nfw}} \lesssim 5$ and $\kappa \propto c_{\mathrm{nfw}}^{1.5}$ for $5 \lesssim c_{\text {nfw }} \lesssim 30$. This clearly proves that the convergence amplitude is sensitive to the halo concentration.

For the number density of source galaxies and the intrinsic ellipticity RMS we are interested in, a raw (un-smoothed) convergence map is very noisy. In order to have a lensing mass map with reasonably small amplitude of the noise, we smooth the convergence map with a Gaussian window function,

$W_{G}(x)=\frac{1}{\pi \theta_{G}^{2}} \exp \left(-\frac{x^{2}}{\theta_{G}^{2}}\right)$.

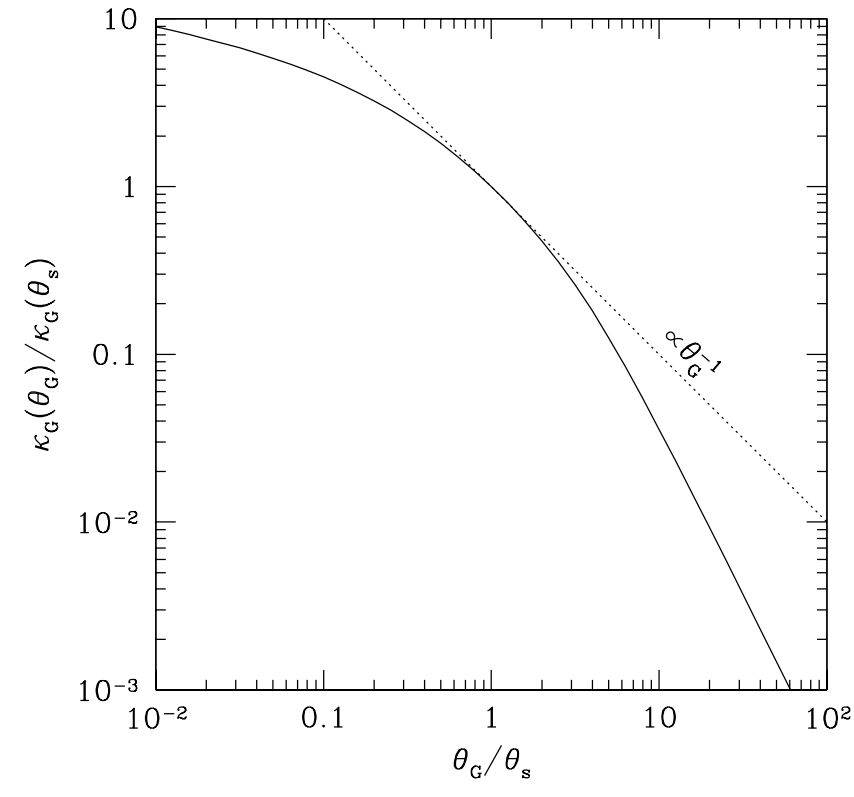

Figure 1. The solid line shows the lensing signal from an NFW halo smoothed with the Gaussian kernel (eq. 13) against the smoothing length. For the concentration parameter, $c_{\mathrm{nfw}}=5$ is adopted here. The dotted line shows scaling of the noise RMS with the smoothing scale $\left(\sigma_{\text {noise }} \propto \theta_{G}^{-1}\right.$, see eq. [12].

The Gaussian smoothing is indeed adopted in actual weak lensing surveys (e.g., Miyazaki et al. 2002). As shown in Van Waerbeke (2000), if the intrinsic ellipticities are uncorrelated between different source galaxies, statistical properties of the resulting noise field can be described by the Gaussian random field theory (Bardeen et al. 1986; Bond \& Efstathiou 1987), on scales where the discreteness effect of source galaxies can be ignored. The Gaussian field is specified by the variance of the noise field that is specified by the number of galaxies contained within a smoothing aperture (Kaiser \& Squires 1993; Van Waerbeke 2000):

$\sigma_{\text {noise }}^{2}=\frac{\sigma_{\epsilon}^{2}}{2} \frac{1}{2 \pi \theta_{G}^{2} n_{g}}$,

where $\sigma_{\epsilon}$ is the RMS amplitude of the intrinsic ellipticity distribution and $n_{g}$ is the number density of source galaxies. Throughout this paper we adopt $\sigma_{\epsilon}=0.4$ and $n_{g}=30 \operatorname{arcmin}^{-2}$, which are typical values for a survey with a limiting magnitude fainter than $R=25.5$ mag in a sub-arcsecond seeing condition. With this choice, the noise RMS $\sigma_{\text {noise }}=0.02 \times\left(1 \mathrm{acrmin} / \theta_{G}\right)$. It is worth noting that $\sigma_{\text {noise }}$ is a direct observable in sense that it can be estimated from a reconstructed weak lensing mass map obtained after randomizing the orientation of source galaxy images.

We need to also take into account the smoothing effect on the lensing signal from a halo. The peak amplitude of the convergence profile of halos is modified by smoothing into

$\kappa_{G}\left(\theta_{G}\right)=\int d^{2} \phi W_{G}\left(\phi ; \theta_{G}\right) \kappa(\phi)$,

where the center of the smoothing kernel is set to the halo center. Hereafter we refer to this peak amplitude as "the lensing signal due to a halo". Figure 1 plots the lensing signal as a function of the smoothing scale. The dotted line shows a scaling of the noise RMS with the smoothing scale $\left(\sigma_{\text {noise }} \propto \theta_{G}^{-1}\right.$ ). The comparison between the solid and dotted curves thus shows how the signalto-noise ratio $(\mathrm{S} / \mathrm{N})$ varies with the smoothing scale. Clearly, an 


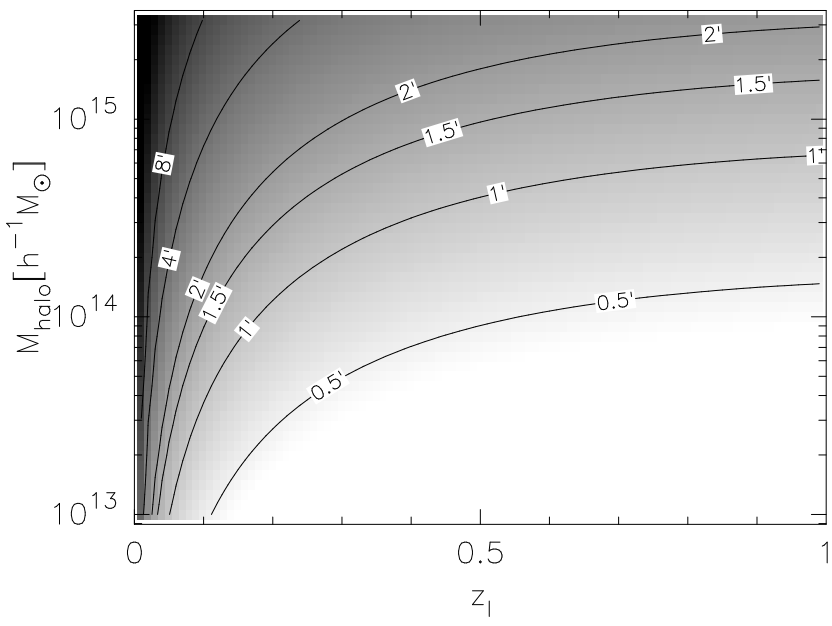

Figure 2. The gray scale with contour lines show the angular scale radius $\theta_{s}$ in unit of arcminutes, in the halo mass $\left(M_{\text {halo }}\right)$ and redshift $\left(z_{l}\right)$ plane. The source redshift is fixed to be $z_{s}=1$.



Figure 3. The gray scale with contour lines shows the $\mathrm{S} / \mathrm{N}$ value for weak lensing halo detection defined by eq. 14. The $\mathrm{S} / \mathrm{N}$ value is computed assuming the universal NFW profile. We adopt, throughout this paper, $\sigma_{\epsilon}=0.4, n_{g}=30 \operatorname{arcmin}^{-1}$ and $\theta_{G}=1$ arcmin.

optimal $\mathrm{S} / \mathrm{N}$ can be attained for $\theta_{G}=(1-2) \times \theta_{s}$. Figure 2 shows the contour plot of $\theta_{s}$ in the halo mass and redshift plane, where the mean mass-concentration relation given by eq. 2] is employed. Note that throughout this paper, the source redshift is fixed to be $z_{s}=1$, which is a typical value for the mean redshift of source galaxies for an actual survey with limiting magnitude $R \simeq 25.5$. Combining Figures 1 and 2 it is suggested that $\theta_{G} \sim 1$ arcmin would be an optimal choice for efficient survey of massive halos with $M_{\text {halo }} \gtrsim 10^{14} h^{-1} M_{\odot}$ at intermediate redshifts.

We define the signal-to-noise ratio, $\mathrm{S} / \mathrm{N}$, for a weak lensing halo detection by ${ }^{1}$

1 Note on the notation issue: Throughout this paper, we denote the $\mathrm{S} / \mathrm{N}$ of weak lensing signals by $\nu$ and distinguish its type by the subscript; $\nu_{\mathrm{nfw}}$ stands for the S/N computed assuming the universal NFW profile, while $\nu_{\text {lens }}$ and $\nu_{\text {noisy }}$ for the $\mathrm{S} / \mathrm{N}$ measured in the noise-free and noisy realizations of numerical weak lensing mock data, respectively (see 3 and 4 .

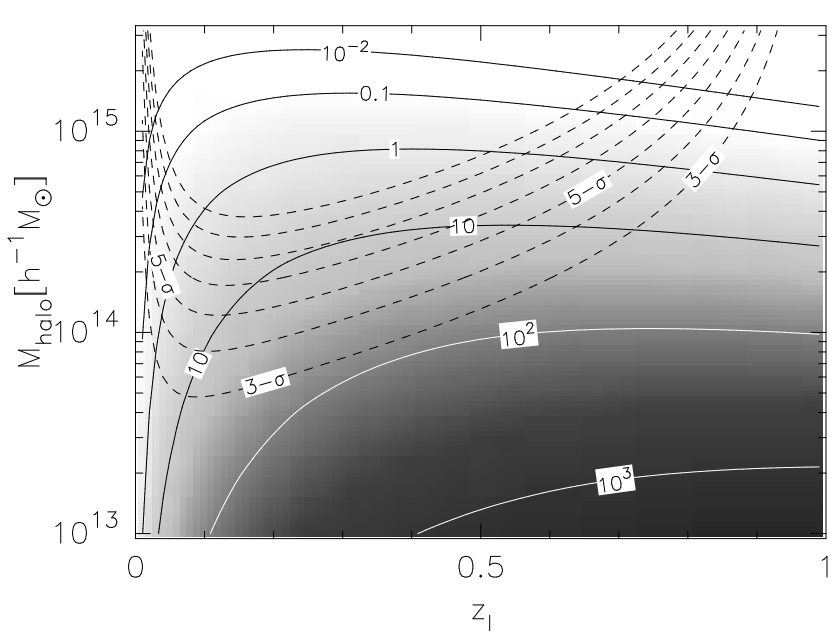

Figure 4. The gray scale with solid contour lines shows the number density of halos per $1 \mathrm{deg}^{2}$ area, a given redshift interval $d z$ and a given logarithmic mass interval $d \log M: d^{2} N_{\text {halo }} / d z / d \log M \equiv$ $d V / d z d n_{\text {halo }} / d \log M$. This is the integrand of eq. 15. The dashed contours show the expected peak height $\nu_{\mathrm{nfw}}$ from 3 to 9 at an interval of $\Delta \nu_{\mathrm{nfw}}=1$. Integrating this number over the peak height above a threshold value $\nu_{\text {th }}$ gives the expected number of peaks per $1 \mathrm{deg}^{2}$ as given by eq. 15 .

$\nu=\frac{\kappa_{G}}{\sigma_{\text {noise }}}$.

Figure 3 shows the contour plot of the $\mathrm{S} / \mathrm{N}$ value computed assuming the universal NFW profile in the halo mass and redshift plane. It is evidently seen from this figure that the lensing signal not only depends on the mass but also on the redshift. This redshift dependence enters mainly through the lensing efficiency function $D_{l} D_{l s} / D_{s}$ (see eqs. [9] and [10]). This makes the mass sensitivity of weak lensing surveys strongly redshift dependent. In other words, the lower mass limit of the detectable halos depends strongly on the redshift. This figure also implies that the effective survey volume of a weak lensing halo search depends on the minimum halo mass that one aims at locating.

It should be noticed that we adopt the mean massconcentration relation to compute the model prediction for the S/N value, though the relation has large scatters (Bullock et al. 2001; Jing 2000). Since the lensing signal, $\kappa_{G}$, depends on the concentration parameter as $\kappa_{G} \propto c_{\mathrm{nfw}}^{1-1.5}$, the scatter likely translates to scatter in the $\kappa_{G}$ value or equivalently in the $\mathrm{S} / \mathrm{N}$. It should be also noted that the lensing signal for a realistic halo is affected by substructures and asphericity of the mass distribution seen in $N$-body simulations. We will carefully investigate these effects, using mock data of weak lensing survey.

\subsection{Model prediction of weak lensing halo counts}

Halos that induce a sufficiently high lensing signal are likely to be identified as peaks in the convergence map, even if the map is contaminated by the noise due to intrinsic ellipticities. Hence, it is convenient to work with the number counts of peaks above a certain threshold value: $N\left(\nu>\nu_{\mathrm{th}} ; \theta_{G}\right)$. In order to compute the number counts, we assume that a high peak is generated by the

We will also use the notations $\nu_{\mathrm{th}}$ and $\nu_{\min }$ to denote a threshold value of the detection and the minimum value to be set (see 4 and $\$$, respectively. 
Table 1. Summary of the weak lensing peak counts computed using eq. 15 for various smoothing scales. Observational parameters adopted are $\sigma_{\epsilon}=$ $0.4, n_{g}=30 \operatorname{arcmin}^{-1}$, and a fixed source redshift of $z_{s}=1$. The second and third columns are for the counts $\nu>5$ and $\nu>4$, respectively.

\begin{tabular}{lcc}
\hline$\theta_{G}$ [arcmin] & $N(\nu>5)\left[1\right.$ sq. deg $\left.{ }^{-1}\right]$ & $N(\nu>4)\left[1\right.$ sq. deg $\left.{ }^{-1}\right]$ \\
\hline 0.5 & 1.47 & 3.38 \\
1 & 2.08 & 4.05 \\
2 & 1.76 & 3.15 \\
3 & 1.30 & 2.27 \\
4 & 0.97 & 1.67 \\
\hline
\end{tabular}

Table 2. Summary of the angular number density (per $1 \mathrm{deg}^{2}$ ) of halos with mass more massive than a given minimum halo mass (the first column). The second and third columns are for the maximum redshifts $z<1$ and $z<0.7$, respectively.

\begin{tabular}{lcc}
\hline Halo mass $\left[h^{-1} M_{\odot}\right]$ & \multicolumn{2}{c}{$N_{\text {halo }}[1$ sq. deg } \\
& $z<1$ & $z<0.7$ \\
\hline$M>5 \times 10^{13}$ & 55 & 30 \\
$M>1 \times 10^{14}$ & 17 & 10 \\
$M>2 \times 10^{14}$ & 4.1 & 2.7 \\
$M>5 \times 10^{14}$ & 0.36 & 0.28 \\
\hline
\end{tabular}

lensing signal from a single massive halo and that the number of false peaks due to noise (i.e., contamination) is much smaller than that of real peaks, as will be shown below in detail. For our fiducial choice of the smoothing scale $\theta_{G}=1$ arcmin, this assumption is reasonably valid for sufficiently high peaks (say $\nu>4$ ), and thus we focus on such high peaks. In this case, the number density of peaks with height greater than a threshold $\nu_{\text {th }}$ per unit solid angle is given by (Kruse \& Schneider 2000; Bartelmann, King \& Schneider 2001)

$$
\begin{aligned}
N\left(\nu>\nu_{\mathrm{th}} ; \theta_{G}, z_{s}\right)= & \int d \chi \frac{d V}{d \chi} \int d M \frac{d n_{\text {halo }}(M, z(\chi))}{d M} \\
& \times \mathcal{H}\left(\nu(M, z)-\nu_{\mathrm{th}}\right),
\end{aligned}
$$

where $\chi$ is the radial comoving distance, $d V / d \chi$ is the comoving volume element per unit solid angle $\left(d V / d \chi=\chi^{2}\right.$ for a flat cosmology), $d n_{\text {halo }} / d M$ is the halo mass function, for which we adopt the fitting function by Sheth \& Tormen (1999), and $\mathcal{H}(x)$ is the Heaviside step function $(\mathcal{H}(x)=1$ for $x>0$ and 0 otherwise). The differential counts are obtained by

$n_{\text {peak }}(\nu)=\left|\frac{d N\left(\nu>\nu_{\mathrm{th}} ; \theta_{G}, z_{s}\right)}{d \nu}\right|$.

Table 1 summarizes the predicted weak lensing peak counts for various smoothing scales. It is shown that a choice of $\theta_{G}=1$ arcmin gives the largest counts, for $\sigma_{\epsilon}=0.4$ and $n_{g}=30 \mathrm{arcmin}^{-2}$. In the following discussion, we will adopt this smoothing scale.

In Figure 4 we plot the selection function of weak lensing halo search with respect to the halo mass and redshift. The gray scale with solid line contours shows the number density of halos per $1 \mathrm{deg}^{2}$ against a given redshift interval of $d z$ and a given logarithmic mass interval of $d \log M$ :

$\frac{d^{2} N_{\text {halo }}}{d z d \log M} \equiv \frac{d V}{d z} \frac{d n_{\text {halo }}}{d \log M}$.

Note that this is the integrand of eq. 15. It is clear that, for a given halo mass, the number density rapidly increases with redshift at

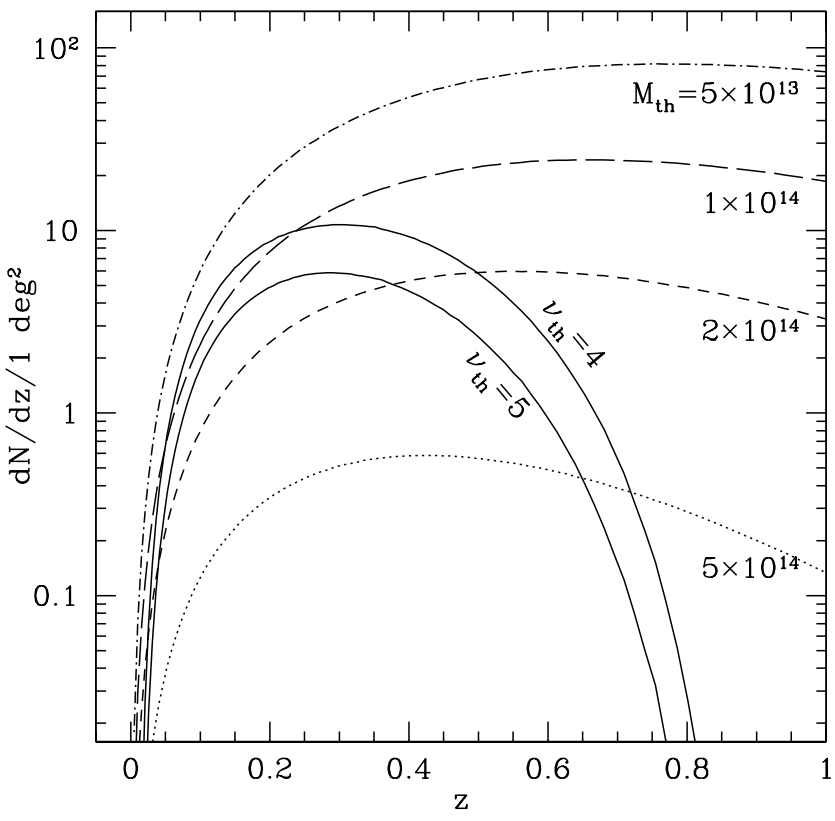

Figure 5. The solid lines show the redshift distribution of halos with a peak height above a threshold value $\nu_{\text {th }}$ per $1 \mathrm{deg}^{2}, d N\left(\nu_{\mathrm{nfw}}>\nu_{\mathrm{th}}\right) / d z / 1$ $\mathrm{deg}^{2}$, computed using eq. 15. The upper and lower solid lines are for $\nu_{\mathrm{th}}=4$ and 5 , respectively. Note that we employed $\theta_{G}=1 \mathrm{arcmin}$ and $z_{s}=1$. The broken lines show the same redshift distributions but for halos with masses above a given minimum mass, $d N_{\text {halo }}\left(M>M_{\mathrm{th}}\right) / d z / 1$ $\operatorname{deg}^{2}$.

low redshifts $z \lesssim 0.2$, and then gradually decreases toward higher redshifts. This is because the volume element, $d V \propto \chi^{2} d \chi$, increases with redshift, whereas the halo number density decreases. The dashed contours show the expected peak height $\nu_{\mathrm{nfw}}$ for the universal NFW halo at intervals of $\Delta \nu_{\mathrm{nfw}}=1$. The comparison between the solid and dashed contours clarifies that, if threshold peak height $\nu_{\text {th }}=4-5$ is employed, the weak lensing halo search is most sensitive to halos that are located at the redshift between $0.1 \lesssim z \lesssim 0.7$ and have masses greater than $10^{14} h^{-1} M_{\odot}$.

Let us look further into the redshift- and mass-dependence of the weak lensing selected halo counts. The solid curves in Figure 5 show the redshift distribution of halos selected by the weak lensing signal. These curves can be compared with the distribution of halos above a certain mass threshold (broken lines). A remarkable difference between these two is evidently seen at redshifts $z>0.5$; the distribution of weak lensing selected halos drops very rapidly, while the mass selected ones decrease very gradually. This is again because the lensing efficiency peaks around $z=0.4$ for source galaxies of $z=1$. Figure 6 shows essentially the same information but from a different angle. The left panel shows the mass function of halos with peak height exceeding a threshold value (the cases of $\nu_{\text {th }}=4$ and 5 are displayed). The lower mass limit in the mass function is determined by the detection threshold, while the decline in the high mass side (which has an approximate exponential slope) is due to the property of the halo mass function (i.e., the exponential shape of its high mass end, see Kruse \& Schneider 2000). Right panels of Figure 6 shows the mass distribution of halos for different redshift intervals as labeled in each plot. The solid (dashed) lines are for halos with $\nu_{\mathrm{nfw}}>5\left(\nu_{\mathrm{nfw}}>4\right)$, while the dotted lines are for all the halos in the corresponding volume. The upper two plots clearly show that, up to $z=0.4$, almost all the halos with $M>2 \times 10^{14} h^{-1} M_{\odot}$ indeed produce high peaks with $\nu_{\mathrm{nfw}}>5$. 


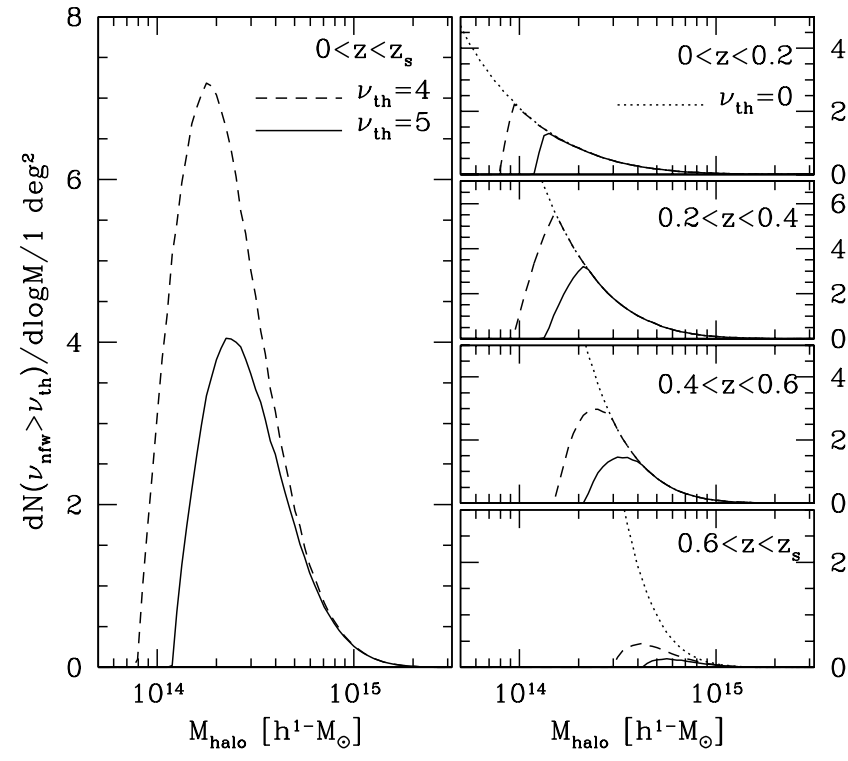

Figure 6. Left Panel: Mass function of halos with $\nu_{\mathrm{nfw}}>\nu_{\mathrm{th}}$ computed using eq. 15. The threshold values are $\nu_{\text {th }}=4$ and 5 for the dashed and solid lines, respectively, The source redshift is taken to be $z_{s}=1$. Right Panels: Same as the left panel but divided into four ranges of the halo redshift as denoted in each plot. The dashed lines show the mass function of halos within the corresponding volume (which is equivalent to set $\nu_{\mathrm{th}}>0$, that is, no limit on the weak lensing signal is imposed). Note that the scales of $y$-axis are labeled on the right side of the plots.

However, for higher redshifts, only very massive halos can produce high peaks.

To summarize the results shown in Figures 4- 6 we argue that weak lensing is not an efficient technique of finding high-redshift halos (say $z>0.6$ ), except for very massive halos of $M>5 \times$ $10^{14} h^{-1} M_{\odot}$, if a mean source redshift is around $z_{s}=1$. It should be noticed that the ability of the weak lensing halo surveys depends on observational conditions. As a survey is deeper, the halo search more enables us to detect less massive halos and halos at higher redshift.

Table 2 gives the number density of halos with mass above a given minimum mass, which is obtained by integrating the halo mass function up to a given maximum redshift. Comparison with Table 1 provides a rough estimate of how weak lensing survey misses mass-selected halos in the given survey volume. A large discrepancy between the halo counts and the weak lensing halo counts is seen, which arises from the redshift dependence of the lensing selection function as shown in Figures 5 and 6

\section{NUMERICAL SIMULATION AND MOCK CATALOGS}

The theoretical model prediction discussed in the previous section provides us with a basic selection function of the weak lensing cluster survey. However, since our model is based on the simple averaged descriptions of the dark matter halos, it does not take into account the individuality of halos which causes a substantial scatter in the lensing signal and may even cause a bias. Furthermore, there are two additional sources of scatter; the projection effect and the intrinsic ellipticity noise. In this section, we will examine these effects in detail using mock numerical experiments.

\section{1 $N$-body simulation}

We used $N$-body data from Very Large $N$-body Simulation (VLS) carried out by the Virgo Consortium (Jenkins et al. 2001, and see also Yoshida et al. 2001 for simulation details). The simulation was performed using a parallel $\mathrm{P}^{3} \mathrm{M}$ code (MacFarland et al. 1998) with a force softening length of $l_{\text {soft }} \sim 30 h^{-1} \mathrm{kpc}$. The simulation employed $512^{3} \mathrm{CDM}$ particles in a cubic box of $479 \mathrm{~h}^{-1} \mathrm{Mpc}$ on a side. It uses a flat cosmological model with $\Omega_{0}=0.3$, $\Omega_{\Lambda}=0.7$, and $h=0.7$. The initial matter power spectrum was computed using CMBFAST (Seljak \& Zaldarriaga 1996) assuming a baryonic matter density of $\Omega_{\mathrm{b}}=0.04$. The normalization of the power spectrum is set by $\sigma_{8}=0.9$. The particle mass $\left(m_{\text {part }}=6.86 \times 10^{10} h^{-1} M_{\odot}\right)$ of the simulation is sufficiently small to guarantee practically no discreteness effect on dark-matter clustering on scales down to the softening length in the redshift range of interest for our purposes (Hamana, Yoshida \& Suto 2002).

\subsection{Weak lensing ray-tracing simulation}

The multiple-lens plane ray-tracing algorithm we used is detailed in Hamana \& Mellier (2001; see also Bartelmann \& Schneider 1992, Jain, Seljak \& White 2000 and Vale \& White 2003 for the theoretical basics and technical issues); here we thus describe only aspects specific to the VLS $N$-body data. In order to generate the density field between $z=0$ and $z \simeq 1$, we use a stack of 6 snapshot outputs from two runs of the $N$-body simulation, which differ only in the realization of the initial fluctuation field. Each cubic box is divided into 4 sub-boxes of $479^{2} \times 119.75 h^{-3} \mathrm{Mpc}^{3}$ with the shorter box side being aligned with the line-of-sight direction. $N$-body particles in each sub-box are projected onto the plane perpendicular to the shorter box side and thus to the line-of-sight direction. In this way, the particle distribution between the observer and $z \simeq 1$ is projected onto 19 lens planes separated equally by $119.75 h^{-1} \mathrm{Mpc}$. Note that in order to minimize the difference in redshift between a lens plane and an output of $N$-body data, only one half of the outputs (i.e. two sub-boxes) from $z=0 N$-body box was used.

The particle distribution on each plane is converted into the surface density field on a $4096^{2}$ regular grid using the triangular shaped cloud (TSC) assignment scheme (Hockney \& Eastwood 1988). $4096^{2}$ grid is chosen to maintain the resolution provided by the $N$-body simulation as well as to remove at the same time the shot noise due to discreteness in the $N$-body simulation. The computation follows the procedure described in Hamana \& Mellier (2001) and Jain et al. (2000).

Having produced surface density fields on all lens planes, we trace $512^{2}$ rays backwards from the observer's point to source plane using the multiple-lens plane algorithm (e.g. Schneider, Ehlers \& Falco 1992). The initial ray directions are set on $512^{2}$ grids with a grid spacing of 0.24 arcmin, and thus the total area covered by the rays is $2.048^{2}$ square degrees. We made 50 realizations of the underlying density field by randomly shifting the simulation boxes in the direction perpendicular to the line-of-sight using the periodic boundary conditions of the simulation ${ }^{2}$. Note that the lens planes coming from the same box are shifted in the same way in order to maintain the clustering of matter in one box.

In the following analyses, we use the lensing convergence map

2 The weak lensing ray-tracing simulation data used in this paper are available from T. Hamana on request, hamanatk@cc.nao.ac.jp. 


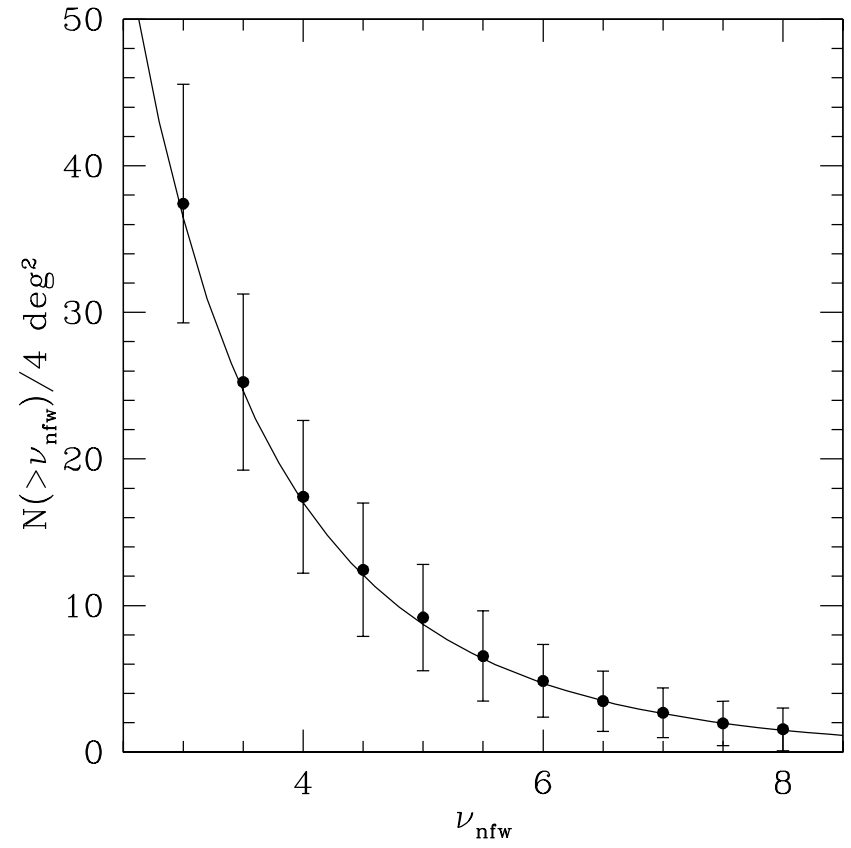

Figure 7. The number counts of the lensing peaks above a given threshold $\nu_{\mathrm{nfw}}$ per a 4 square degrees area. The solid curve shows the model prediction computed from eq. (15), while the filled circles with error bars denote the mean and RMS among 50 realizations of mock simulation data. Note that the peak height of a halo is estimated from the halo mass and redshift in the manner described in the text 3.4 .

of the source plane closest to $z=1$. This is, we call, the noise-free lensing map.

\subsection{Adding noise}

To simulate a more realistic lensing survey, we add noises due to intrinsic ellipticities of source galaxies to the simulated lensing map. Actual observations are further affected by other systematics such as imperfect conditions of telescope and/or of electric devices of detector. Examining these effects is beyond the scope of the present paper, and thus we do not consider such observational effects.

As extensively shown in Van Waerbeke (2000), the noise field due to the intrinsic ellipticities is well approximated by the Gaussian random field on angular scales of our interest, if the ellipticities are uncorrelated between different sources (see also Jain \& Van Waerbeke 2000). The Gaussian noise added to our $\kappa$ map has variance

$\sigma_{p i x}^{2}=\frac{\sigma_{\epsilon}^{2}}{2} \frac{1}{n_{g} \theta_{p i x}^{2}}$

where $\theta_{\text {pix }}=0.24$ arcmin is the pixel size of our $\kappa$ maps. The noise RMS on pixels is $\sigma_{p i x}=0.22$ which is much larger than the RMS of the lensing convergence field.

\subsection{Halo catalogs on the light-cone}

We identify dark matter halos in 5 low redshift outputs of the $N$-body simulation using the standard friends-of-friends algorithm with a linking parameter of $b=0.164$ (in units of the mean particle separation). Halos with mass greater than $1 \times 10^{13} h^{-1} M_{\odot}$, which corresponds to the mass of 146 simulation particles, are used in the

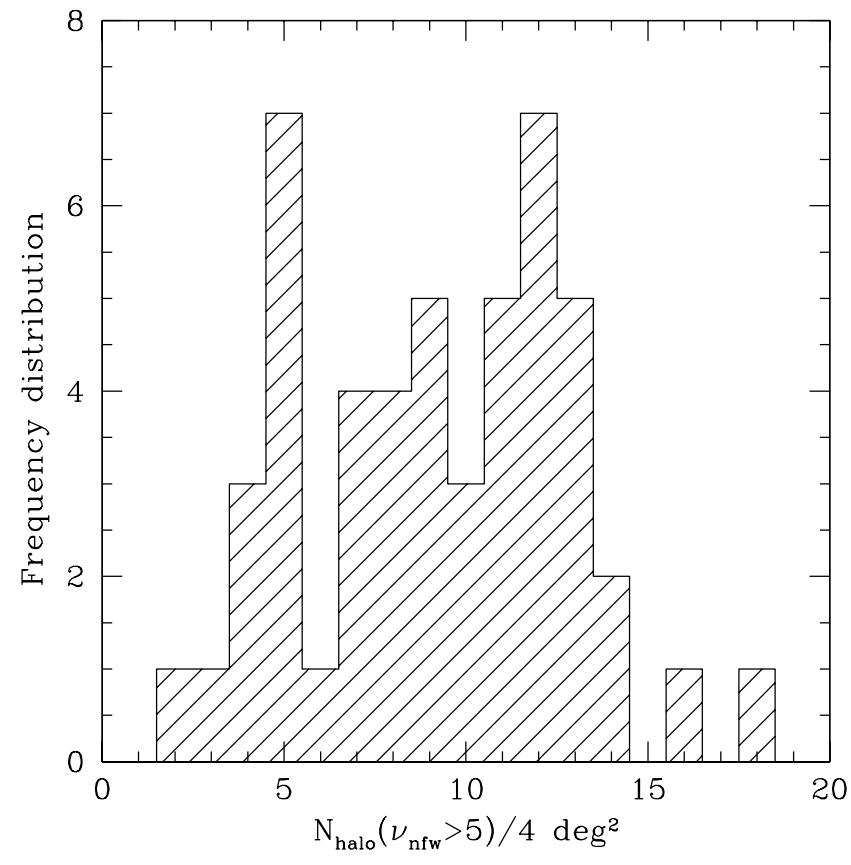

Figure 8. The frequency distribution of numbers of halos with the expected peak height (see $\$ 3.4$ for its definition) $\nu_{\mathrm{nfw}}>5$ from 50 realizations of 4 square degrees.

analyses below. We define the halo center to be the center-of-mass position of member particles.

In order to evaluate the halo shape, we compute the inertia tensor of the mass distribution;

$$
\begin{aligned}
I_{i j} & =\int d \boldsymbol{x}^{3}\left(x_{i}-\bar{x}_{i}\right)\left(x_{j}-\bar{x}_{j}\right) \rho(\boldsymbol{x}) \\
& =m_{p} \sum_{k=1}^{N_{p}}\left(x_{i, k}-\bar{x}_{i}\right)\left(x_{j, k}-\bar{x}_{j}\right),
\end{aligned}
$$

where $i, j$ run from 1 to $3, \bar{x}_{i}$ denotes the center-of-mass position and $N_{p}$ is the number of member particles of the halo. From the inertia tensor we compute the eigen vectors and convert them to the axial ratios $a / c$ and $a / b(a \leqslant b \leqslant c)$. We also compute the trace of the inertia tensor,

$Q \equiv \frac{1}{3}\left(I_{11}+I_{22}+I_{33}\right)$.

This is compared with the expectation value for the NFW halo,

$$
\begin{aligned}
Q_{\mathrm{NFW}} & =\frac{4 \pi}{3} \int_{0}^{r_{\mathrm{vir}}} d r r^{4} \rho_{\mathrm{nfw}}(r) \\
& =M_{\mathrm{vir}} r_{\mathrm{vir}}^{2} q\left(c_{\mathrm{nfw}}\right),
\end{aligned}
$$

with

$q\left(c_{\mathrm{nfw}}\right)=\frac{c_{\mathrm{nfw}}^{3}-3 c_{\mathrm{nfw}}^{2}-6 c_{\mathrm{nfw}}+6\left(1+c_{\mathrm{nfw}}\right) \log \left(1+c_{\mathrm{nfw}}\right)}{6 c_{\mathrm{nfw}}^{2}\left[\left(1+c_{\mathrm{nfw}}\right) \log \left(1+c_{\mathrm{nfw}}\right)-c_{\mathrm{nfw}}\right]}$.

Note that $q\left(c_{\mathrm{nfw}}\right)$ can be approximated by $q\left(c_{\mathrm{nfw}}\right) \simeq 0.22 c_{\mathrm{nfw}}^{-0.26}$ for $3 \lesssim c_{\text {nfw }} \lesssim 30$. The ratio $Q / Q_{\text {NFW }}$ gives an estimate of the concentration of the mass distribution. We also define the following quantity as an estimate of the line-of-sight elongation of the halo mass distribution:

$R=\frac{2 I_{33}}{I_{11}+I_{22}}$ 


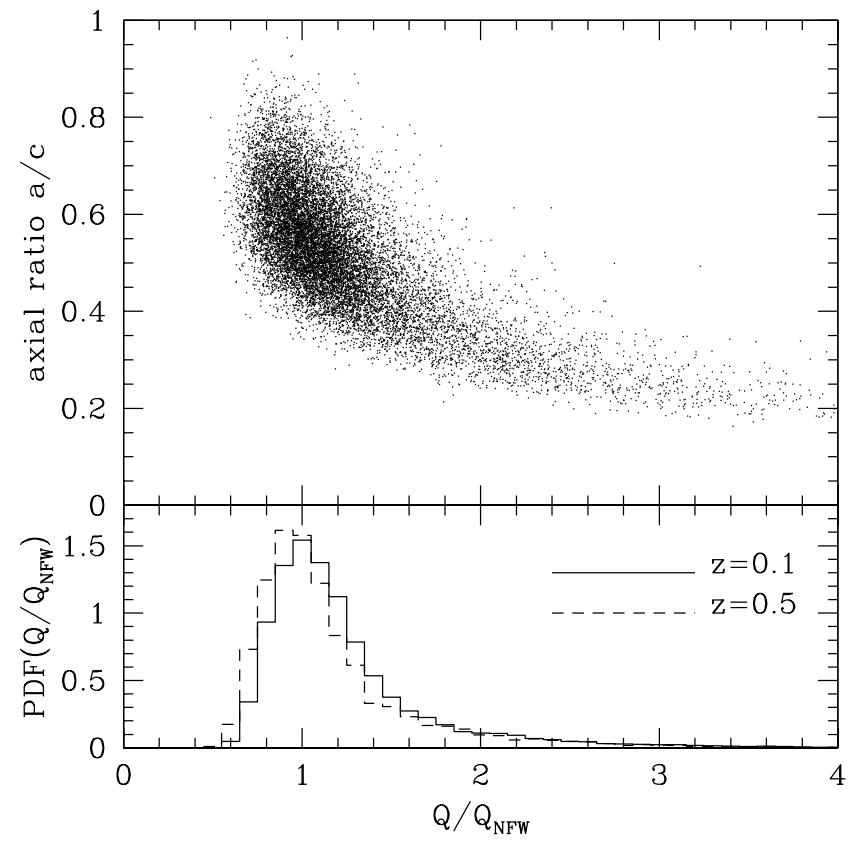

Figure 9. Upper Panel: The scatter plot showing the relation between $Q / Q_{\text {NFW }}$ and the axial ratio $a / c$ for halos with $M>3 \times 10^{13} h^{-1} M_{\odot}$ in the $z=0.1$ output. Here $Q_{\mathrm{NFW}}$ is defined by eq. 21) and is computed using the relation eq. 4. Lower Panel: The PDF of $Q / Q_{\mathrm{NFW}}$ of halos with $M>3 \times 10^{13} h^{-1} M_{\odot}$, the solid and dashed lines for $z=0.1$ and $z=0.5$ outputs, respectively.

where $x_{3}$ is taken by the direction parallel to the line-of-sight. Although this is rather a crude estimation, $R>1$ or $R<1$ generally implies halos elongated along or perpendicular to the line-of-sight.

Halo catalogs on the light-cone are generated by stacking the simulation outputs in the same manner as in the ray-tracing experiments. We use the 50 realizations which have the same underlying matter distribution as used in the ray-tracing data. This combined data set allows us to directly relate the distribution of dark matter halos to the weak lensing convergence map.

In summary, each halo in the mock catalogs has data on the mass, the estimates of the halo shape $Q$ and $R$ defined by eqs. 20 and 23, respectively, the redshift computed from the radial distance to a halo, and the angular position converted from the spatial position. In addition, for each halo, we compute the virial radius and the concentration parameter using eqs. (4) and (2), from the halo mass and redshift of $N$-body data output time (which are $z=0,0.1,0.3,0.5$ and 0.8 ). These values allow us to evaluate the expected lensing signal using eq. [13, with eq. [8, which is correct only if the halo has the NFW profile. Similarly, we also compute $Q_{\text {NFW }}$ using eq. 21. Moreover, we introduce a flag parameter for each halo, to quantify the following possible problem that arises from our use of multiple-lens plane ray-tracing algorithm. As a result of projection of $N$-body particles into 4 lens planes, member particles of given one halo located close to the boundary could be separated into different planes. Such a split halo would generate an artificially deformed (or even separated) lensing signal in a $\kappa$ map. We assign $f l a g=1$ for such halos whose virial radius is larger than the distance between the halo center and the closest plane, flag $=2$ for halos whose scale radius is larger than the distance to the closest plane, and flag $=0$ otherwise. The fraction of flagged halos is very small, $0.88 \%$ for flag $=1$ and $0.19 \%$ for flag $=2$, thus it does not have significant influence on our analyses below,

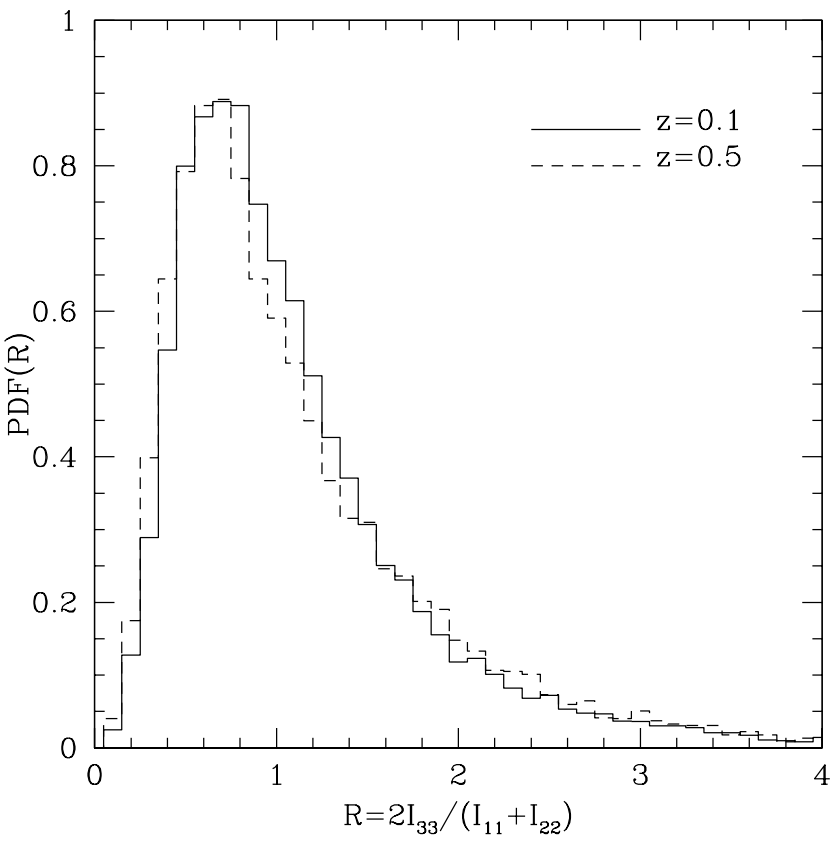

Figure 10. The PDF of the $R$ defined by eq. 23 for halos with $M>$ $3 \times 10^{13} h^{-1} M_{\odot}$ at $z=0.1$ (solid line) and $z=0.5$ (dashed line) outputs. Note that the median of the distributions is $\sim 0.92$, and $55 \%$ of the all samples has $R<1$.

but we carefully check if flagged halos cause any artificial eccentric signals in the lensing convergence map.

The expected lensing signal of each halo is converted into the peak height in the same way as in eq. 14, which we denote as $\nu_{\mathrm{nfw}}$. Total numbers of halos with $\nu_{\mathrm{nfw}}>5$ and $\nu_{\mathrm{nfw}}>4$ in our 50 realizations are 459 and 871 , respectively, which are sufficiently large to allow the statistical analyses of weak lensing halos.

The redshift and mass distributions of halos in the halo catalog agree well with the model predictions in 2.2 Figure 7 plots the accumulate number counts of halos against the expected peak height, $\nu_{\mathrm{nfw}}$. The solid curve is the theoretical prediction of eq. 15 and agrees well with the simulation result. This agreement is not surprising, because ingredients of the theoretical model (the universal profile and halo mass function) are calibrated by $N$-body simulations.

The counts of halos with $\nu_{\mathrm{nfw}}>5$ in $2.048^{2}$ square degrees is found to be $N\left(\nu_{\mathrm{nfw}}>5\right)=9.18 \pm 3.63$, where the error represents the RMS among 50 realizations. This is in good agreement with the theoretical prediction of 8.71. However, the RMS is somewhat large and slightly larger than the Poisson fluctuation. Figure 8 shows the distribution of numbers of halos with $\nu_{\mathrm{nfw}}>5$ in each realization, quite a wide spread is evidently seen. This is mainly due to the very strong clustering of massive halos. Accordingly, given a limited survey area, the halo counts may significantly vary from survey to survey.

The lower panel of Figure 9 shows the PDFs of $Q / Q_{\mathrm{NFW}}$ computed from massive halos with $M>3 \times 10^{13} h^{-1} M_{\odot}\left(Q_{\mathrm{NFW}}\right.$ is computed using the mean mass-concentration relation eq. [2]). The PDF peaks at $Q / Q_{\mathrm{NFW}} \sim 1$ as expected, but has rather broad distribution. No significant difference in the PDFs between the five different redshift boxes was found. The upper panel shows the scatter plot in $Q / Q_{\mathrm{NFW}}-a / c$ plane for halos with $M>$ $3 \times 10^{13} h^{-1} M_{\odot}$ from $z=0.1$ output. It is clear that the mass concentration defined by $Q / Q_{\mathrm{NFW}}$ correlates with the axial ratio, 


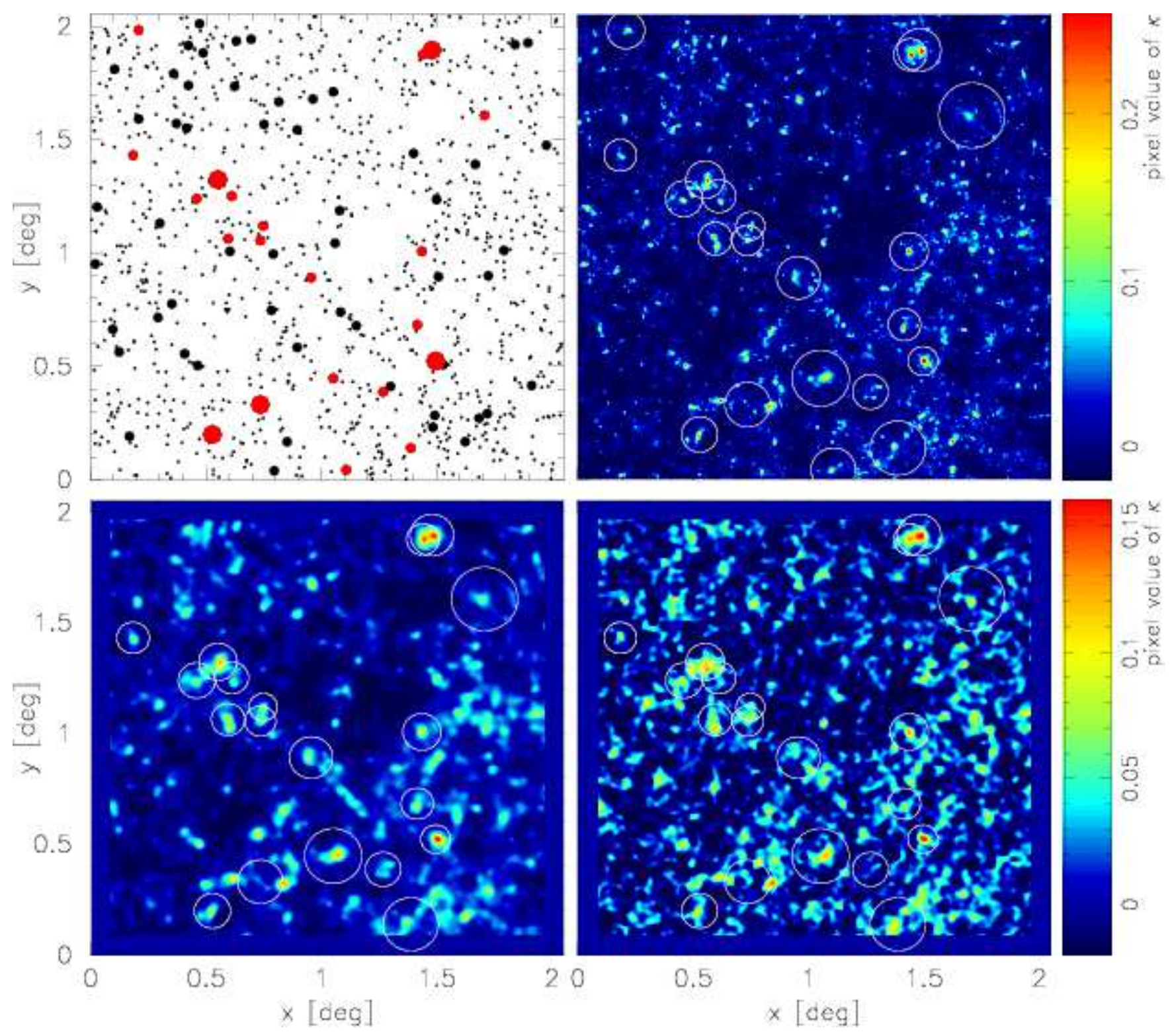

Figure 11. Upper-left: The celestial distribution of halos identified from one realization of mock light-cone data generated stacking $N$-body outputs. The large filled circles denote halos with $M_{\text {halo }}\left[h^{-1} M_{\odot}\right]>3 \times 10^{14}$, the small filled circles are for $3 \times 10^{14}>M_{\text {halo }}\left[h^{-1} M_{\odot}\right]>8 \times 10^{13}$ and the dots are for $8 \times 10^{13}>M_{\text {halo }}\left[h^{-1} M_{\odot}\right]>1 \times 10^{13}$. Only the halos within redshift interval between 0.1 and 0.7 are displayed. Upper-right: The gray map shows the lensing convergence map on the $512^{2}$ pixels with the pixel size of 0.24 arcmin obtained from ray-tracing experiment through the same mass distribution as used in the upper-left panel. The circles show the positions of halos with $\nu_{\mathrm{nfw}}>4$, the center of which is set at the position of each halo and the radius of which shows the projected virial radius (see $\$$.3.4 for details). Bottom-left: Same as the top-left panel but the convergence map is smoothed by the Gaussian window function with $\theta_{G}=1 \mathrm{arcmin}$. The edge regions of the field with $5 \mathrm{arcmin}$ width are masked because of incomplete smoothing due to the lack of $\kappa$ data on outer region. The gray scale of this plot is same as that of the bottom-right panel. Bottom-right: Same as the bottom-left panel but the Gaussian noise is added before smoothing is applied. From comparison with the other plots, it is clear that high peaks due to massive halos are still identifiable.

which implies that an extended mass distribution is likely associated with eccentric mass distributions such as halos having substructures or on-going merger. We note that the PDF of axial ratio peaks at $a / c \sim 0.5$ and thus most of halos significantly deviate from the spherical symmetry. This broad distribution leads to significant scatter in the relation between the expected and observed lensing signals as seen below.

Figure 10 shows the PDF of the shape parameter $R$ defined by eq. 23. for massive halos with $M>3 \times 10^{13} h^{-1} M_{\odot}$. A broad distribution is evidently seen and we checked that the distribution does not largely change over the redshift range we considered $(z<0.8)$. It is important to note that the median of the distribution is less than unity $(\sim 0.92)$, thus more than half of the massive halos (specifically $55 \%$ for halos with $M>3 \times 10^{13} h^{-1} M_{\odot}$ irrespective of the redshift) have $R<1$. This is a natural consequence of the random orientation of the halos' major axis; among the spatial three directions, it is more likely that the major axis of a halo is elongated along either of two directions perpendicular to the line-ofsight rather than along it. The skewed distribution leads to system- 
atic bias in the relation between the expected and observed lensing signals (see 5.2 and Appendix C for details).

\subsection{Visual impressions}

Figure 11 represents the lensing convergence maps and the celestial distribution of halos, which are taken from one of the 50 realizations. In the top-right panel, the gray scale shows $\kappa$ value on the $512^{2}$ pixels with the pixel size of 0.24 arcmin. The bottom-left panel shows the same convergence map as in the top-right panel but after smoothed by the Gaussian filter function of $\theta_{G}=1$ arcmin. Adding the Gaussian noise yields the noisy map shown in the bottom-right panel. While the noise indeed alters the appearance of the convergence map, the very high peaks remain identifiable.

The top-left panel shows the distribution of all the halos identified by the FOF scheme in the original $N$-body simulations from which the convergence maps in the other plots are generated. Note that the size of point scales with halo masses. A good correspondence in positions between massive halos and high lensing signals on the $\kappa$ map is seen. This correspondence is more clearly seen if halos are selected by means of the expected $\nu_{\mathrm{nfw}}$ value: in the convergence maps (the top-right and two bottom panels), the positions of halos with $\nu_{\mathrm{nfw}}>4$ are marked by the circles with their centers being placed at the halo center-of-mass position, and their radii show the angular virial radius (see 3.4. The improvement in the correspondence reflects the fact that the lensing signal is determined by combined contributions of the halo mass, the redshift and the halo concentration, rather by the mass alone.

\section{RESULTS ON PEAK COUNTS}

In this section, we study the number counts of high peaks in the lensing convergence map. To do this, we use three kinds of $\kappa$ maps; (i) the noise-free $\kappa$ maps, (ii) the noisy $\kappa$ maps where the Gaussian noise was added in the manner described in 3.3 and (iii) the pure noise maps without the cosmological lensing signals. All these maps are smoothed with a Gaussian window function with $\theta_{G}=1$ $\operatorname{arcmin}($ eq. 11]). We identify positive (negative) curvature peaks in the maps as pixels that have higher (lower) values of $\kappa$ than all the surrounding eight pixels (Jain \& Van Waerbeke 2000, and also see Miyazaki et al 2002 for the first application to actual observational data). The peak height is defined as the ratio of the convergence value at the peak to the noise RMS, as given by eq. 14. For all the three cases, $\sigma_{\text {noise }}=0.02\left(\sigma_{\epsilon}=0.4\right.$ and $\left.n_{g}=30 \operatorname{arcmin}^{-2}\right)$ is adopted.

\subsection{The peak PDF}

The probability distribution function (PDF) of peaks in the convergence map is studied in detail by Jain \& Van Waerbeke (2000). Here we briefly summarize the characteristics of the peak PDF that are relevant for the subsequent discussion.

Figure 12 shows the peak PDFs in the noise-free $\kappa$ (dashed histogram), the noisy $\kappa$ map (solid histogram) and the pure noise map (dotted histogram). The upper panel shows the sum of the positive and negative curvature peaks, while the lower panel shows the contribution from the positive peaks only. In the absence of the noise, the peak PDF reflects the characteristics of the nonlinear gravitational clustering. Most of negative peaks are due to the negative curvature peaks (troughs). There is a cutoff in the negative peak height because the mass density contrast cannot be lower than

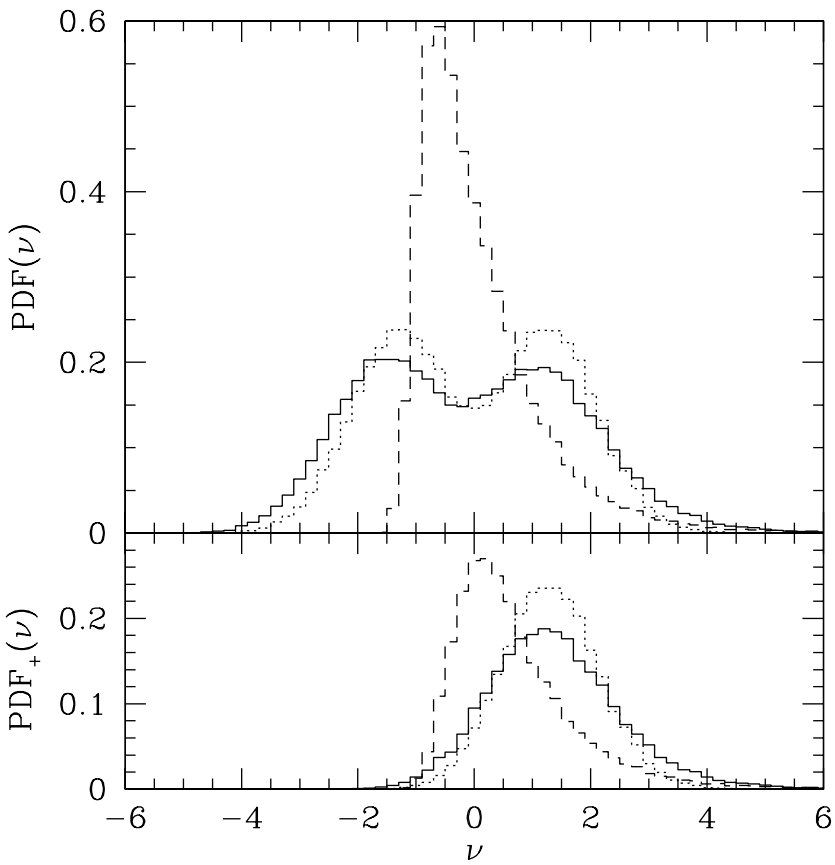

Figure 12. The simulation results for the PDFs of the peaks in the noisefree $\kappa$ (dashed histogram), in the noisy $\kappa$ map (solid histogram) and in the pure noise map (dotted histogram). Shown is the cumulative results from the 50 realizations. The upper panel shows the sum of the positive and negative curvature peaks, while the lower panel shows the contribution from the positive peaks only. In the total $50 \times 2.048^{2}$ square degrees' area, there are $59,908,107,165$ and 114,250 peaks for the noise-free $\kappa$, the noisy $\kappa$ map and the pure noise map, respectively. The corresponding numbers for positive curvature peaks are $31,043,53,123$, and 57,141.

-1 . One can also find a tail toward the high positive peak height which arises from the lensing signals due to massive halos (Kruse $\&$ Schneider 2000). Another noticeable feature seen from the lower panel is that some of the positive curvature peaks have a negative peak height, which occurs when the peak due to a halo is superposed on a void region in the same line-of-sight. If the noise field is Gaussian, the PDF from the pure-noise map is derivable from the the Gaussian random field theory (Bardeen et al. 1986; Bond \& Efstathiou 1987; Van Waerbeke 2000). The symmetric bimodal feature of the peak PDF is due to the superposition of two contributions from peaks with positive and negative curvatures. While adding the noise to the cosmological $\kappa$ map alters the peak PDF drastically, there remain some characteristic features that contain useful cosmological information. First, the asymmetric double peaks are due to the skewed feature of the cosmological $\kappa$ PDF as stated above. Second, there are excesses over the noise PDF on both high positive $(\nu>3)$ and negative peaks $(\nu<-3)$. These features were indeed detected in the recent weak lensing survey by Miyazaki et al. (2002).

It is worth pointing out that the pure noise PDF can be directly estimated from an actual observed data. First, we can generate a pure noise map by applying mass-reconstruction to a randomized catalog where the orientation of source galaxy images is randomized (to remove coherent image distortions from lensing signals). Then we can measure the peak PDF from the pure noise map, as was performed in Miyazaki et al. (2002). This allows one to estimate the contamination rate (the false peak rate) directly from the data. 


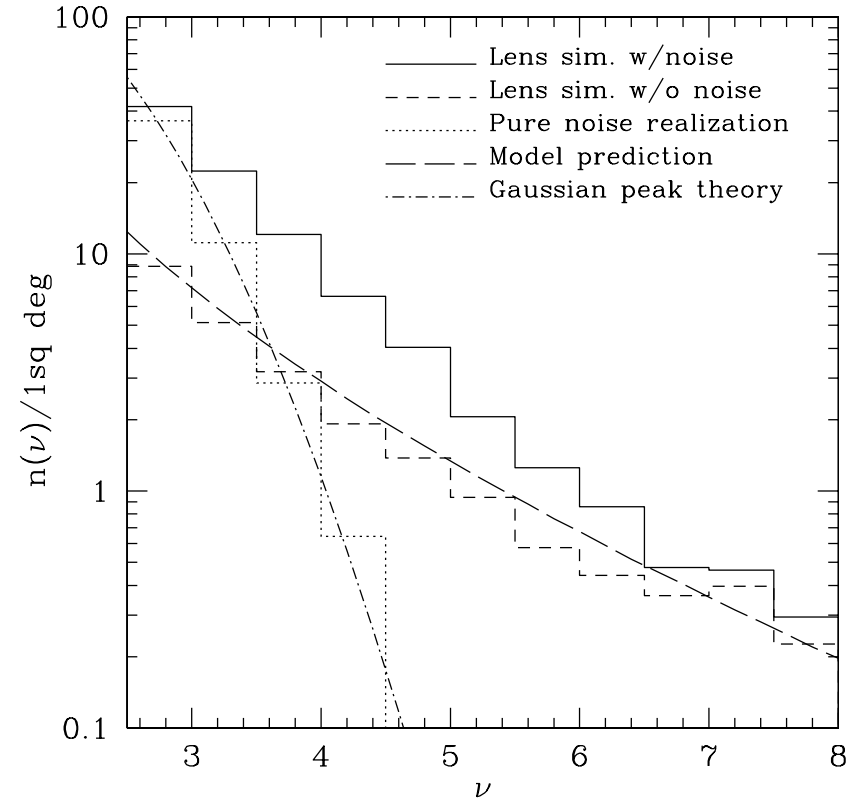

Figure 13. Counts of peaks per 1 square degrees. The histograms are from the numerical experiments; the noisy $\kappa$ maps (solid), the noise-free $\kappa$ maps (dashed) and the pure noise realizations (dotted). The long-dashed line shows the model prediction, eq. 16 with eq. [15], and the dot-dashed line shows the prediction by Gaussian random field theory (e.g., Bond \& Efstathiou 1987; Van Waerbeke 2000).

\subsection{High peak counts}

In the following, we restrict our discussion to the number counts of high peaks that are mainly due to massive halos. Figure 13 shows the number density of (positive curvature) peaks per 1 square degrees. The histograms show the average values computed from the 50 realizations of the numerical experiments; the noisy $\kappa$ maps (solid), the noise-free $\kappa$ maps (dashed) and the pure noise maps (dotted). The long-dashed line shows the model prediction from eq. 16, while the dot-dashed line shows the prediction for the pure noise counts (Van Waerbeke 2000). From comparison of the results for the lensing maps with and without the noise, it is clear that the noise significantly increases the peak counts over a range of peak heights we have considered. Even high peaks with $\nu>5$ are similarly boosted, while there is no such event in the pure noise counts, as shown by the dotted curve. There are two main reasons for the enhancement (see $\$ 5.3$ and Appendix $[\mathrm{B}$ for further details). First, addition of the noise leads to modifications of the peak height as well as of the peak position. Second, the noise generates spurious peaks in the noisy maps. In particular, the latter effect is likely to show up in a biased way depending on the peak height. Since the convergence field around a given high peak has greater amplitude than the average field, adding the noise onto such a region is more likely to induce spurious high peaks around the real peak. We develop a correction scheme to the model peak counts in Appendix [B] The corrected counts are in reasonable agreement with the simulation result for high peaks $\nu>5$.

\section{RESULTS ON THE CORRESPONDENCE BETWEEN HALOS AND PEAKS}

In this section, we examine correspondences between massive halos and peaks in the weak lensing convergence maps, using the re-
Table 3. Classification of matching relations between halos and peaks. The double-lined arrows denote the primary match, while the single-lined arrows denote the secondary match. The last two columns summarize the number of halos/peaks in each class, the third (fourth) column is for matching between the halo catalog and the peak list from the noise-free (noisy) $\kappa$ map. Note that halos with $\nu_{\mathrm{nfw}}>3$ (856 halos in total) and peaks with $\nu_{\text {lens }}>3$ (669 peaks) and $\nu_{\text {noisy }}>3$ (2417 peaks) are considered.

\begin{tabular}{lccc}
\hline class & matching relation & \multicolumn{2}{c}{ number of objects } \\
& & $\nu_{\mathrm{nfw}}-\nu_{\text {lens }}$ & $\nu_{\mathrm{nfw}}-\nu_{\text {noisy }}$ \\
\hline i & halo $\rightleftharpoons$ peak & 475 & 624 \\
ii & halo with no paired peak & 367 & 222 \\
iii & peak with no paired halo & 177 & 1637 \\
iv & halo $\rightleftharpoons$ peak & 14 & 10 \\
v & halo $\rightleftarrows$ peak & 17 & 156 \\
\hline
\end{tabular}

sults of our ray-tracing experiments and the halo catalogs created from the $N$-body simulation outputs. In doing so, we follow a realistic procedure which is to be used in weak lensing surveys: we focus on high peaks above a certain threshold in the lensing map as strong candidates for halos. The usefulness of the weak lensing halo search can be quantified in terms of completeness and efficiency. As will be shown later in detail, choosing a threshold $\nu_{t h} \simeq 4-5$ gives an optimal balance between the efficiency and completeness. We will also carefully examine if there is a correlation between the detectability of halos and their shape, because it could produce a bias in halo catalogs constructed from the weak lensing method.

In order to make realistic weak lensing survey catalogs, we impose a criterion on the field selection of simulations so that no nearby massive halo is included in the target fields. Specifically, we discard simulated fields if there is a halo (or possibly more) with $M_{\text {halo }}>10^{14} h^{-1} M_{\odot}$ at $z<0.1$. Similar criterion, such as no known nearby X-ray luminous cluster of galaxies or no nearby optically identified massive cluster, is usually imposed on the field selection of an actual observation, because such a nearby massive cluster occupies a large portion of a survey field and makes a distant halo search difficult and inefficient. 31 out of the 50 simulated fields pass this criterion. In addition, we discard one field in which there is a massive halo with $\nu_{\mathrm{nfw}}>5$ having flag $=2$ (see 3.4 for a description of $(\mathrm{lag})^{3}$. We analyze the remaining 30 fields. We note that we do not use data within 5 arcmin of the edge of each simulated field because of the incomplete smoothing due to the lack of data at the outer region. The total field size is then 106 square degrees.

\subsection{Matching halos with peaks in lensing mass maps}

We shall now examine relations between the peak height in weak lensing maps and the properties of halos through the expected peak height for the NFW halos $\left(\nu_{\mathrm{nfw}}\right.$, see $\$ 2.1$. We restrict our discussion to halos with $\nu_{\mathrm{nfw}}>3$ only. The halo catalogs contain 856 such halos in total. On the other hand, the ray-tracing simulations directly enable us to find peaks in the $\kappa$ maps. We consider two kinds of the $\kappa$ maps; those with and without the noise due to the intrinsic ellipticities. The peaks in the two maps are identified in the manner described in $\$ 4$ The noise-free maps contain 669 peaks with $\nu_{\text {lens }}>3$, while the noisy maps contain 2417 peaks with

\footnotetext{
3 Including this field in our analysis below does not change the conclusions, but we decided to discard it for having conclusive results.
} 
$\nu_{\text {noisy }}>3$. Thus, the noise significantly enhances the peak counts. It is also worth noting that the 669 peaks in the noise-free maps is significantly smaller than the expected number of peaks from the halo catalogs. We will carefully investigate this discrepancy in the subsequent sections.

We consider halos and peaks with $\nu>3$ only in order to avoid mismatches between peaks and physically unrelated halos. Because of large number density of low mass halos, including such halos rapidly increases the chance of mis-matching. This is especially the case when one attempts to match peaks in the noisy $\kappa$ maps with halos because false peaks due to the noise can be accidentally matched with physically unrelated halos. The criterion $\nu>3$ is chosen based on the fact that most of such high peaks are expected to be caused by real halos, even when the lensing map is contaminated by the noise (see Figure 13. Also the criterion value is reasonably lower than the observational threshold value which is generally set by $\nu \gtrsim 4$. This guarantees most halos of interest to us are included in the final halo catalog.

We carry out the peak-halo matching in two directions: whether a peak has a corresponding halo in the halo catalog, and whether a halo has a counter-peak in the simulated $\kappa$ map. Note that the peak position does not necessarily agree with the halo's center-of-mass position exactly and this is more likely the case for halos that have substructures or are in the merger process. For this reason, we search for a matched pair candidate within a radius of 12 pixels ( 2.88 arcmin) from the peak position or from the halo center. This maximum angular separation 2.88 arcmin is chosen so that it is larger than the smoothing radius of $\theta_{G}=1$ arcmin, while it is still smaller than the angular virial radius of a massive halo at $0.1 \lesssim z \lesssim 0.7$. If there are more than one pair candidate within the radius, we take the closest one as the primary candidate and consider the others as the secondary candidate.

We classify the peak-halo matching into the following five classes: Class-(i) is that the both directions agree; both a halo and a peak are their primary pair candidate. Class-(ii) is defined as halos that have no paired peak within a radius of 12 pixels from the halo center. We will call such halos as "missing halos". Class-(iii) is defined as peaks with no paired halo within a radius of 12 pixels from the peak position. We will often refer to such peaks as "false peaks". Class-(iv) denotes a halo that has a matched peak candidate, but is not the primary matched halo of that peak. Class-(v), which is the opposite to the class-(iv), is that a peak has a matched halo candidate but is not the primary matched peak of that halo. The resulting numbers for the noise-free and noisy maps are summarized in Table 3

Typical properties of halos or peaks in the class-(ii-v) are explained as follows. The class-(ii) halos are those which have a peak height lower than the criterion value $(\nu=3)$ though the expected peak height, $\nu_{\mathrm{nfw}}$, exceeds it. This is mainly due to departures from the NFW profile or/and the projection effect. In the class-(iii) peaks there are two kinds: The first is the peaks which have corresponding halos but have the expected peak heights, $\nu_{\mathrm{nfw}}$, lower than the criterion value, though the measured peak heights in a $\kappa$ map exceed it. This is again due to departures from the NFW profile or/and the projection effect. The other is the peaks which do not have corresponding halos but are generated by the noise. The former accounts for most of the class-(iii) peaks in noise-free $\kappa$ maps, while the latter accounts for a large fraction of those in noisy $\kappa$ maps. The class(iv) halos are due to the projection effect; if two or more halos are located at different redshifts along almost the same line-of-sight, after smoothing of the $\kappa$ map only one peak appears, because the smoothing merges those into one structure. Finally, in the class-(v)

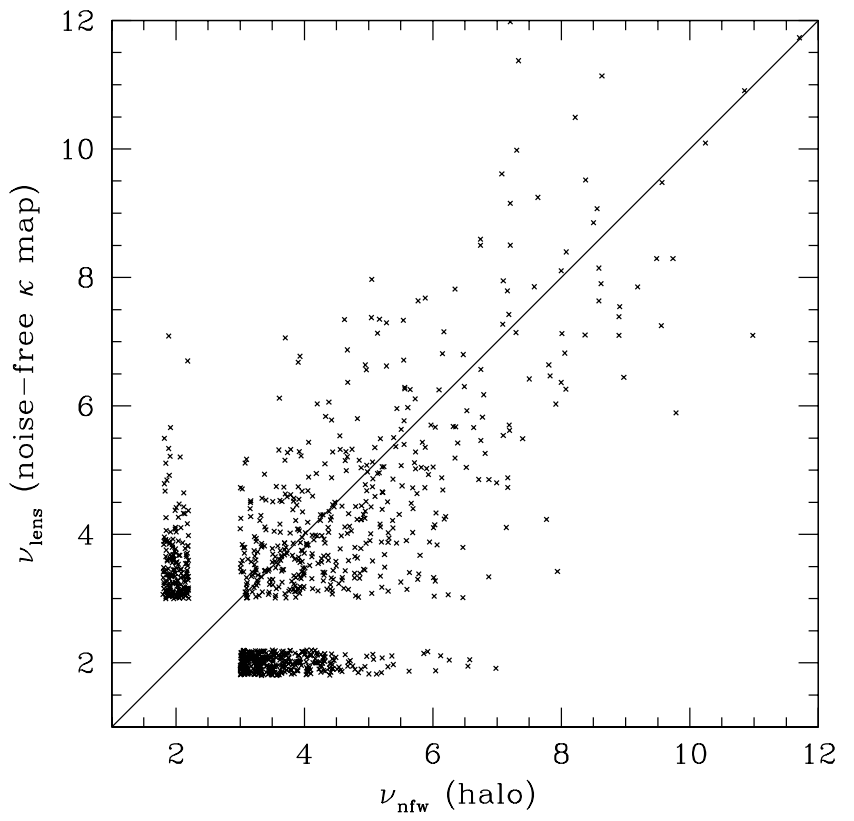

Figure 14. The correspondences between halos and peaks identified in the noise-free $\kappa$ maps in $\nu_{\mathrm{nfw}}-\nu_{\text {lens }}$ plane. The class-(i-iii) objects are shown. The class-(ii) objects are placed within a narrow track around $\nu_{\text {lens }} \sim 2$, regardless of their true value, and the class-(iii) objects are placed at $\nu_{\mathrm{nfw}} \sim$ 2 as well.

peaks there are two kinds: The first is the peaks due to substructures within a halo. This accounts for most of the class-(v) peaks in noise-free $\kappa$ maps. The other is false peaks due to the noise generated around the primary peak due the massive halo. Because the convergence field around the high peak is biased and thus larger than the average field, adding the noise into such region is more likely to generate false peaks with a relatively high peak height. This accounts for most of the class-(v) peaks in noisy $\kappa$ maps.

In the rest of this section, we consider the class-(i-iii) objects only, and do not deal with the class-(iv) and (v) objects, because the class-(iv) and (v) objects are the secondary matched pairs associated with the primary matched pair in the vicinity. This roughly mimics the actual observation procedure in which the secondary objects are investigated in detail in course of a follow-up observation of the primary matched object.

\subsection{Halos versus peaks in noise-free $\kappa$ maps}

Let us first look into the correspondence between high peaks identified in the noise-free $\kappa$ maps ( $\left.\nu_{\text {lens }}\right)$ and properties of halos through the expected peak height $\left(\nu_{\mathrm{nfw}}\right)$. The main purpose of this subsection is to examine the amplitude of the irreducible scatter in the relation induced by both the individuality of halo's mass distribution and the projection effect.

Figure 14 shows the distribution of the class-(i-iii) objects in the $\nu_{\mathrm{nfw}}-\nu_{\text {lens }}$ plane. Since the class-(ii) halos do not have a matched peak with height $\nu_{\text {lens }}>3$ in the $\kappa$ map (although the halos have a high expected peak height of $\nu_{\mathrm{nfw}}>3$ ), such halos are placed in a narrow locus around $\nu_{\text {lens }}=2$ for illustrative purpose. For the similar reason, the class-(iii) peaks are placed around $\nu_{\mathrm{nfw}}=2$. The distribution of the class-(i) halos shows a large scatter around the ideal relation $\nu_{\text {lens }}=\nu_{\mathrm{nfw}}$ (shown by the solid line in the upper panel) over the range of $\nu$ we consider. It is worth noting that the distribution for a fixed $\nu_{\mathrm{nfw}}$ value is slightly biased 


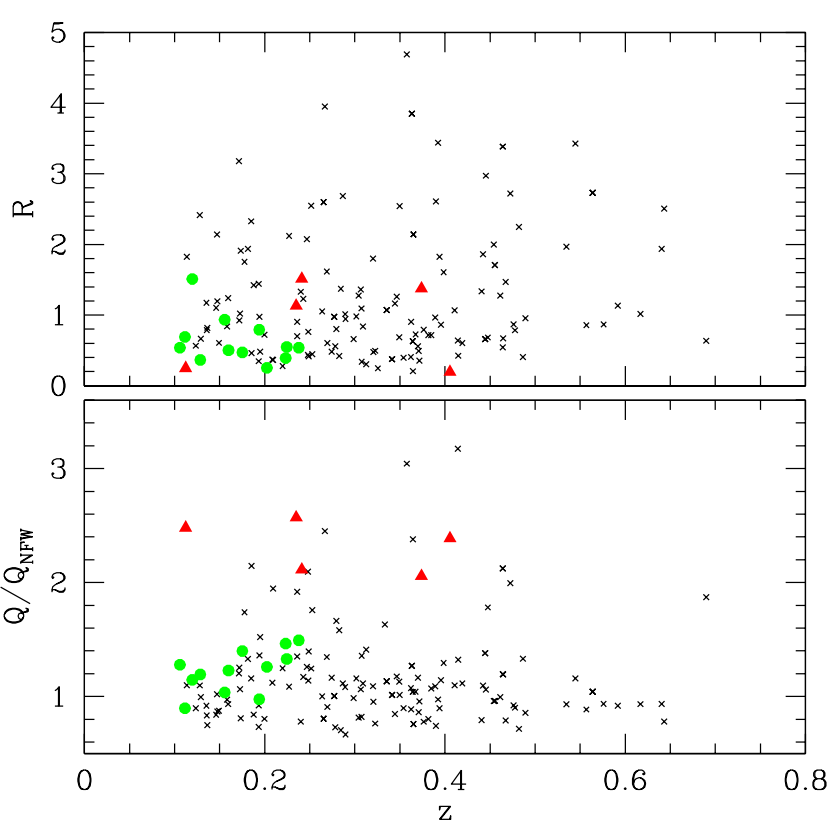

Figure 15. The scatter plot for the distribution of the halos in $z-Q / Q_{\mathrm{NFW}}$ plane (lower panel) and in $z-R$ plane (upper panel). The filled circle and triangle symbols are for the class-(ii) halos with $\nu_{\mathrm{nfw}}>5$. For comparison, the class-(i) halos with $5<\nu_{\mathrm{nfw}}<7.5$ are also plotted by the cross symbols. It is apparent from the lower panel that the class-(ii) halos can be separated into two subclasses: those with $Q / Q_{\mathrm{NFW}}>2$ are plotted by the filled triangles, while the rests are plotted by the filled circles.

toward lower $\nu_{\text {lens }}$ values. The mean and RMS of the differences, $\nu_{\text {lens }}-\nu_{\mathrm{nfw}}$, are -0.24 and 1.2 , respectively. Since no noise is added to the $\kappa$ map in this case, the scatter and bias must be ascribed to the two effects; (1) the mass distribution within a halo is not spherical nor completely universal, causing deviations of the peak heights from the expected values computed from our simple model, and (2) a chance projection of structures along the same line-of-sight alters the lensing signal due to the halo. For the former, although we have so far used the average relation for the halo concentration, given by eq. (2), it was shown in Jing (2000) that the halo concentrations among halos with a given mass obey the $\log$-normal distribution with the variance $\sigma\left(\ln c_{\text {nfw }}\right) \approx 0.2$. This roughly corresponds to a $20 \%$ RMS scatter in the $c_{\mathrm{nfw}}$ relation. Given the approximate relation $\kappa \propto c_{\mathrm{nfw}}^{1-1.5}$ for the peak height due to a massive halo (see 2], the RMS scatter in the $c_{\mathrm{nfw}}$ distribution translates into $20-30 \%$ RMS scatters in the lensing signal $\nu_{\mathrm{nfw}}$ and therefore equivalently in the $\nu_{\text {lens }}-\nu_{\text {nfw }}$ relation. For example, for halos with $\nu_{\mathrm{nfw}}=5$, the RMS of $\sigma_{\nu} \sim 1$ is caused. Second, the lensing projection due to physically unrelated structures along the same line-of-sight sight of a halo also contributes to the scatter in $\nu_{\text {lens. }}$. Following Hoekstra (2001), this scatter can be estimated from the cosmic weak lensing variance under the assumption that the halo and the large-scale structures are uncorrelated. For the Gaussian smoothing scale $\theta_{G}=1^{\prime}$, the amplitude of the (linear) convergence variance is $\sigma_{\kappa}\left(1^{\prime}\right)=0.01$ for the $\Lambda \mathrm{CDM}$ model, corresponding to the RMS deviation of $\sigma_{\nu}=0.5$ because of the noise $\sigma_{\text {noise }}\left(1^{\prime}\right)=0.02$ (see eq. 12]). Hence, these two effects can basically explain the amplitude of the scatter in the $\nu_{\text {lens }}-\nu_{\mathrm{nfw}}$ relation in Figure 14

We also argue that the systematic bias in the $\nu_{\text {lens }}-\nu_{\text {nfw }}$ relation can be explained by the following two effects. The first is the fact that more than half of the halos have $R<1$ as discussed in

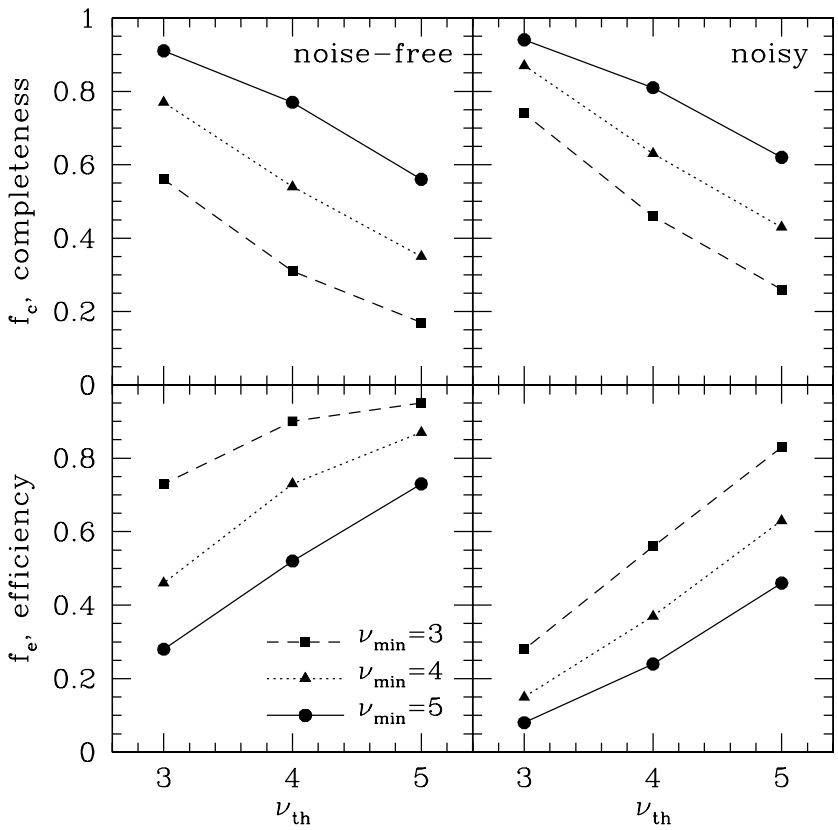

Figure 16. Upper Panels: The completeness $f_{c}\left(\nu>\nu_{\mathrm{th}} \mid \nu_{\mathrm{nfw}}>\nu_{\min }\right)$ defined by eq. 24], as a function of the threshold peak height $\nu_{\mathrm{th}}$. Three cases for the minimum expected peak value, $\nu_{\min }=3,4$ and 5 are shown by the filled squares, triangles and circles, respectively. Lower Panels: The efficiency $f_{e}\left(\nu_{\mathrm{nfw}}>\nu_{\mathrm{min}} \mid \nu>\nu_{\mathrm{th}}\right)$ defined by eq. 25], as a function of the threshold peak height $\nu_{\mathrm{th}}$. The left panels are for the noise-free case $\left(\nu=\nu_{\text {lens }}\right)$, while the right panels are for the noisy case $\left(\nu=\nu_{\text {noisy }}\right)$.

3.4 and Figure 10 Since $R$ positively correlates with $\nu_{\text {lens }} / \nu_{\text {nfw }}$ (see Figure C2 in Appendix C), it causes the bias. The second is the projection with a void region in the same line-of-sight which reduces the lensing signal (see Metzler et al. 2001; White et al. 2002; Padmanabhan et al 2003 for detailed study on the projection effect).

Let us turn to the class-(ii) halos in Figure 14 Among the 367 class-(ii) halos, there are 17 halos that are supposed to produce peaks with $\nu>5$ in the $\kappa$ maps if the halos have the NFW profile (i.e., $\nu_{\mathrm{nfw}}>5$ in our notation). Why are these halos missing? Figure 15 explains this in terms of deviation of the mass distribution within a halo from the spherically symmetric NFW profile. We again use the parameters $Q$ and $R$ to quantify the mass distribution within a halo (see equations (20) and (23), respectively): $Q / Q_{\mathrm{NFW}}<1(>1)$ characterizes a halo that has centrally more (less) concentrated mass distribution than expected from the NFW profile, while $R>1(<1)$ describes that the mass distribution is elongated along (perpendicular) to the line-of-sight ( $R=1$ for the spherical symmetric halo). Therefore, a halo with $Q / Q_{\mathrm{NFW}}>1$ and/or $R<1$ likely leads to a smaller lensing signal than expected from the NFW profile (see Appendix $[$ for more detailed analysis of the relations between the halo shape and the amplitude of the lensing signal). The upper panel of Figure 15 shows the distribution of the class-(ii) halos in $z-Q / Q_{\mathrm{NFW}}$ plane, while the lower panel shows the distribution in $z-R$ plane. For comparison, the figure also shows the distributions for the class-(i) halos with $5<\nu_{\text {nfw }}<7.5$ by the cross symbols. From the lower panel, it is clear that the class-(ii) halos can be divided into two distinct subgroups; those which have $Q / Q_{\mathrm{NFW}}>2$ (plotted by the filled triangles) or $Q / Q_{\mathrm{NFW}}<2$ (plotted by the filled circles). We indeed find that halos with $Q / Q_{\mathrm{NFW}}>2$ show clear signatures of substructures or on-going merging, resulting in the extended mass dis- 




Figure 17. The scatter plot for the halos in the redshift-halo mass plane. The contours show the expected peak height $\nu_{\mathrm{nfw}}$ from 3 to 9 at an interval of $\Delta \nu_{\mathrm{nfw}}=1$. The class-(i) halos matched with peaks identified in noise-free $\kappa$ maps are plotted in the upper-left $\left(\nu_{\text {lens }}>5\right)$, upper-right $\left(5>\nu_{\text {lens }}>4\right)$ and lower-left panels $\left(4>\nu_{\text {lens }}>3\right.$ ). The lower-right panel shows the scatter plot for the class-(ii) "missing halos".

tribution (the $\kappa$ map of such halos is shown in Figure A1 for example). The remaining objects with $Q / Q_{\mathrm{NFW}}<2$ tend to be located at a low redshift $(z<0.25)$, where the lensing efficiency is relatively small for the source redshift $z_{s}=1$ we consider, and have a small $R$, mostly $R<1$. The examples are shown in Figures $\mathrm{A} 2$ and $\mathrm{A3}$ These halos typically have prominent substructures and the whole mass distribution within the halo is indeed elongated along the perpendicular direction to the line-of-sight. Except for such irregular systems, almost all massive halos with $\nu_{\mathrm{nfw}}>5$ generate a high peak with $\nu_{\text {lens }}>3$. It is important to note that the distribution of the class-(ii) halos in Figure 15 is not clearly distinguished from that of the class-(i) halos, reflecting the fact that the lensing signal is also affected by the projection effect.

Turn next to the class-(iii) objects in Figure 14 which are the peaks with $\nu_{\text {lens }} \geqslant 3$ in the lensing map, but do not have the corresponding halos in the halo catalog (contains only halos with $\nu_{\mathrm{nfw}}>3$ ). Among the class-(iii) objects there are 8 events with $\nu_{\text {lens }}>5$ which we call "false peaks". We carefully looked into each case in the $\kappa$ map and searched for a possible related halo in the halo catalog. We found that the 5 events are associated with substructure of massive halos or the secondary local peaks of merger systems (note that the peaks are separated from the primary peak of the halo center by more than our searching distance, 12 pixels $=2.88$ arcmin). Figure A4 shows the $\kappa$ map of one of such cases. The remaining 3 peaks are due to a chance projection of two moderately massive halos at different redshifts (Figure A5 shows the one example). Therefore, except for the three chance pro- jection systems, the false peaks with $\nu_{\text {lens }}>5$ are all associated with a real massive halo as the secondary peak.

In order to quantify the ability of weak lensing survey to locate massive halos, we consider the completeness and efficiency. We define the completeness by the fraction of halos, which are detected as high peaks above a given threshold $\nu_{\text {th }}$ in the $\kappa$ map, relative to all the halos with the expected lensing signal, $\nu_{\mathrm{nfw}}$, greater than a given minimum value $\nu_{\min }$ :

$f_{c}\left(\nu_{\text {lens }}>\nu_{\text {th }} \mid \nu_{\text {nfw }}>\nu_{\text {min }}\right)=\frac{N_{\mathrm{i}}\left(\nu_{\text {lens }}>\nu_{\text {th }} \mid \nu_{\text {nfw }}>\nu_{\text {min }}\right)}{N_{\mathrm{i}+\mathrm{ii}}\left(\nu_{\mathrm{nfw}}>\nu_{\mathrm{min}}\right)}$,

where $N_{\mathrm{i}+\mathrm{ii}}\left(\nu_{\mathrm{nfw}}>\nu_{\mathrm{min}}\right)$ denotes the number of halos with $\nu_{\mathrm{nfw}}>\nu_{\text {min }}$ which, in our classification, corresponds to the number of the class-(i) and (ii) such halos, and $N_{\mathrm{i}}\left(\nu_{\text {lens }}>\nu_{\text {th }} \mid \nu_{\mathrm{nfw}}>\right.$ $\left.\nu_{\text {min }}\right)$ denotes the halos having $\nu_{\mathrm{nfw}}>\nu_{\min }$ matched with the peaks with $\nu_{\text {lens }}>\nu_{\text {th }}$ (thus these are the class-(i) objects). Similarly, we define the efficiency by the fraction of peaks, which are associated with halos with the expected peak height exceeding a given minimum value $\nu_{\min }$, relative to all the peaks with height greater than a given threshold $\nu_{\mathrm{th}}$.

$f_{e}\left(\nu_{\mathrm{nfw}}>\nu_{\mathrm{min}} \mid \nu_{\text {lens }}>\nu_{\mathrm{th}}\right)=\frac{N_{\mathrm{i}}\left(\nu_{\mathrm{nfw}}>\nu_{\mathrm{min}} \mid \nu_{\text {lens }}>\nu_{\mathrm{th}}\right)}{N_{\mathrm{i}+\mathrm{iii}}\left(\nu_{\text {lens }}>\nu_{\mathrm{th}}\right)}$,

where $N_{\mathrm{i}+\mathrm{iii}}\left(\nu_{\text {lens }}>\nu_{\mathrm{th}}\right)$ denotes the number of peaks with $\nu_{\text {lens }}>\nu_{\text {th }}$ which corresponds to the number of the class-(i) and (iii) such peaks.

The results are plotted in the left panels of Figure 16 as a function of $\nu_{\text {th }}$. The upper panels show the completeness $\left(f_{c}\right)$ and the 


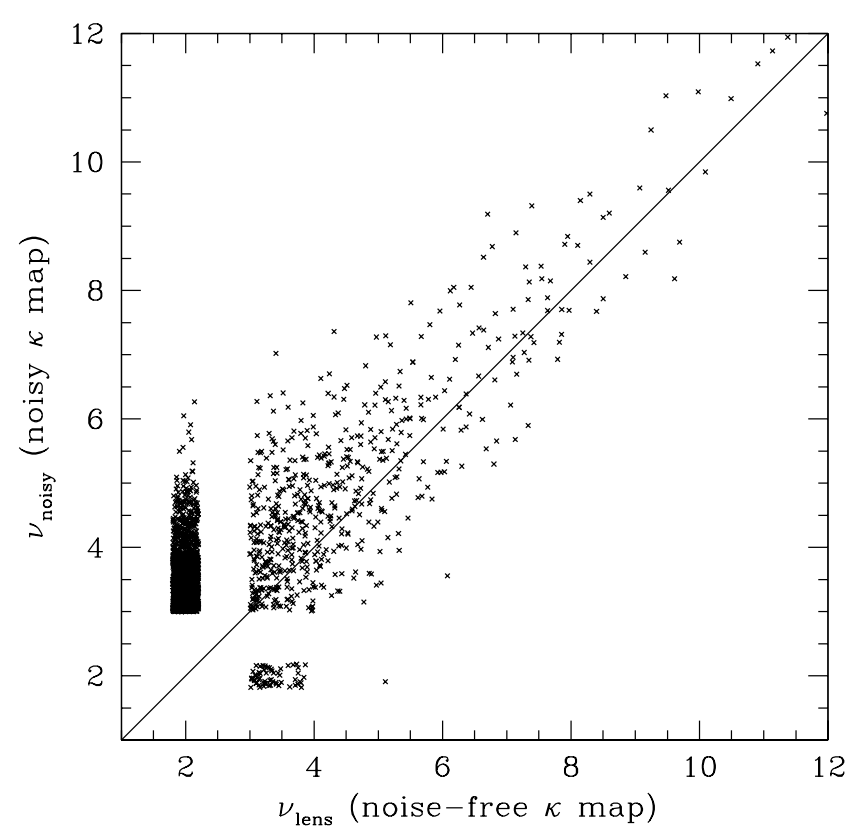

Figure 18. The correspondences between the peak heights identified in the noise-free and noisy $\kappa$ maps. The peaks, which have $\nu_{\text {lens }}>3$ but are missed in the noisy $\kappa$ map or have $\nu_{\text {noisy }}<3$, are placed within a narrow locus at $\nu_{\text {noisy }} \sim 2$, regardless of their true $\nu_{\text {noisy }}$ value. Similarly, the peaks, which have $\nu_{\text {noisy }}>3$ but no paired peak with $\nu_{\text {lens }}>3$, are placed at $\nu_{\text {lens }} \sim 2$,

lower panels show the efficiency $\left(f_{e}\right)$. As expected, these two estimates behave inversely, that is, when $f_{e}$ goes up, $f_{c}$ goes down. Therefore the optimal choice of the $\nu_{\text {th }}$ value should be determined by balance between the two estimates, which we discuss in the latter section with taking into account effects of the noise.

The plot for the completeness shows that only about half of halos with $\nu_{\mathrm{nfw}}>5$ produce peaks with $\nu_{\text {lens }}>5$ in the noisefree $\kappa$ maps, and that 77 percent of the halos have $\nu_{\text {lens }}>4$. The completeness becomes worse if $\nu_{\mathrm{th}} \gtrsim \nu_{\mathrm{min}}$, as expected. It is also shown that a good completeness ( sets $\nu_{\text {th }} \lesssim \nu_{\text {min }}-1$. This can be understood from the fact that the RMS scatter caused by the individuality of halos and the projection effect is $\sigma_{\nu} \sim 1.2$. Note that some fraction of the halos with $\nu_{\text {lens }}$ much smaller than the expected peak heights $\nu_{\mathrm{nfw}}$ are irregular systems or un-relaxed systems, and therefore the completeness is likely to be improved for samples of regular, well relaxed halos. It should be also noted that the noise increases the completeness (see 5.4 and the upper right panel).

Let us turn to the efficiency. Since no noise is added to the $\kappa$ maps, the efficiency given here should be regarded as an estimate for an ideal lensing survey. Clearly, the efficiency is very high. If one sets the detection threshold to be $\nu_{\text {th }}=5,73$ percent of all the peaks correspond to real halos with $\nu_{\mathrm{nfw}}>5$, and 95 percent for $\nu_{\mathrm{nfw}}>3$. Even for $\nu_{\mathrm{th}}=4$ the efficiency is still high, $90 \%$ for $\nu_{\mathrm{nfw}}>3$. Therefore, in the absence of the noise, the contamination rate (the fraction of false peaks) is very small for detection threshold $\nu_{\text {th }}>4$.

Figure 17 shows the scatter plot of the class-(i) and -(ii) halos in the redshift-halo mass plane. The contours show tracks of the expected peak height $\nu_{\mathrm{nfw}}$ from 3 to 9 at an interval of $\Delta \nu_{\mathrm{nfw}}=1$. The class-(i) halos matched with peaks in noise-free $\kappa$ maps are plotted in the upper-left $\left(\nu_{\text {lens }}>5\right)$, upper-right $\left(5>\nu_{\text {lens }}>4\right)$ and lower-left panels $\left(4>\nu_{\text {lens }}>3\right)$, respectively. The lower-right

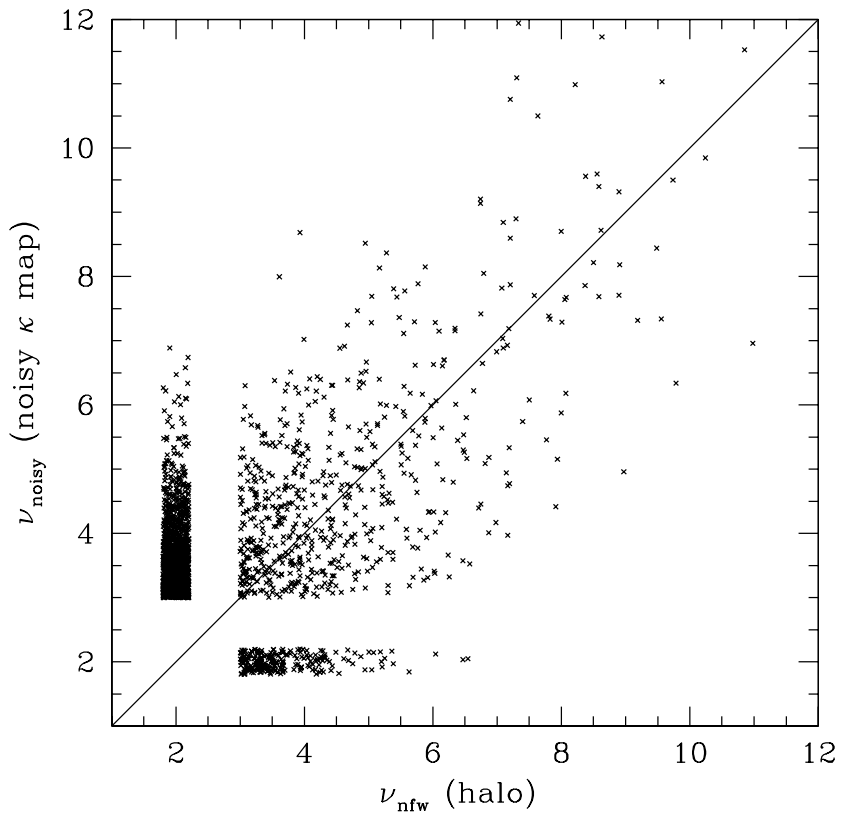

Figure 19. Same as Figure 14 but for the correspondence between halos and peaks identified in the noisy $\kappa$ maps.

panel shows the scatter plot for the class-(ii) halos, namely " missing halos". Importantly, halos within a small range of the peak height are spread over a wide range of masses due to the projection effect and the individuality of the halo mass distribution.

\subsection{Effects of the noise on the lensing peaks}

Before examining correspondences between peaks identified in the noisy $\kappa$ maps and halos in the halo catalog, we quantitatively examine how the noise alters the peaks due to halos and generates false peaks in the lensing map.

Matching between the noise-free peaks and the noisy peaks was done in the same manner described in 5.1 In what follows, the peaks classified in the class-(i-iii) (its analogy to $\nu_{\text {lens }}-\nu_{\text {noisy }}$ correspondence) are considered. Again, we only deal with peaks with $\nu>3$.

Figure 18 shows the relations between $\nu_{\text {lens }}$ and $\nu_{\text {noisy }}$ plotted in the same manner as in Figure 14 We emphasize that the statistical properties of the $\nu_{\text {lens }}-\nu_{\text {noisy }}$ relation do not follow the Gaussian distribution, even though the Gaussian noise is added. The reason is as follows. The noise not only alters the peak height from massive halos but also changes the peak position. For example, if a large positive noise is accidentally added on a neighboring pixel around the original peak position, the pixel could be identified as a peak that is even higher than the original peak. Therefore, the distribution of $\nu_{\text {noisy }}$ 's is not simply expressed as the convolution of that of $\nu_{\text {lens }}$ 's with the Gaussian noise, but the $\nu_{\text {lens }}-\nu_{\text {noisy }}$ relation becomes asymmetric and is biased toward large $\nu_{\text {noisy }}$ values. This explains the asymmetric $\nu_{\text {lens }}-\nu_{\text {noisy }}$ relation clearly shown in Figure 18 The mean and RMS of the differences, $\nu_{\text {noisy }}-\nu_{\text {lens }}$, among the class-(i) objects with $\nu_{\text {lens }}>4$ (this restriction is imposed in order to avoid an incomplete sampling of low $\nu_{\text {noisy }}$ pairs) are 0.31 and 0.90 , respectively. Therefore the peak heights of massive halos identified in a noisy $\kappa$ map are, on average, boosted by the noise. This bias partly accounts for the excess in the peak number counts over the simple theoretical prediction, eq. 16, as discussed in 4.2 


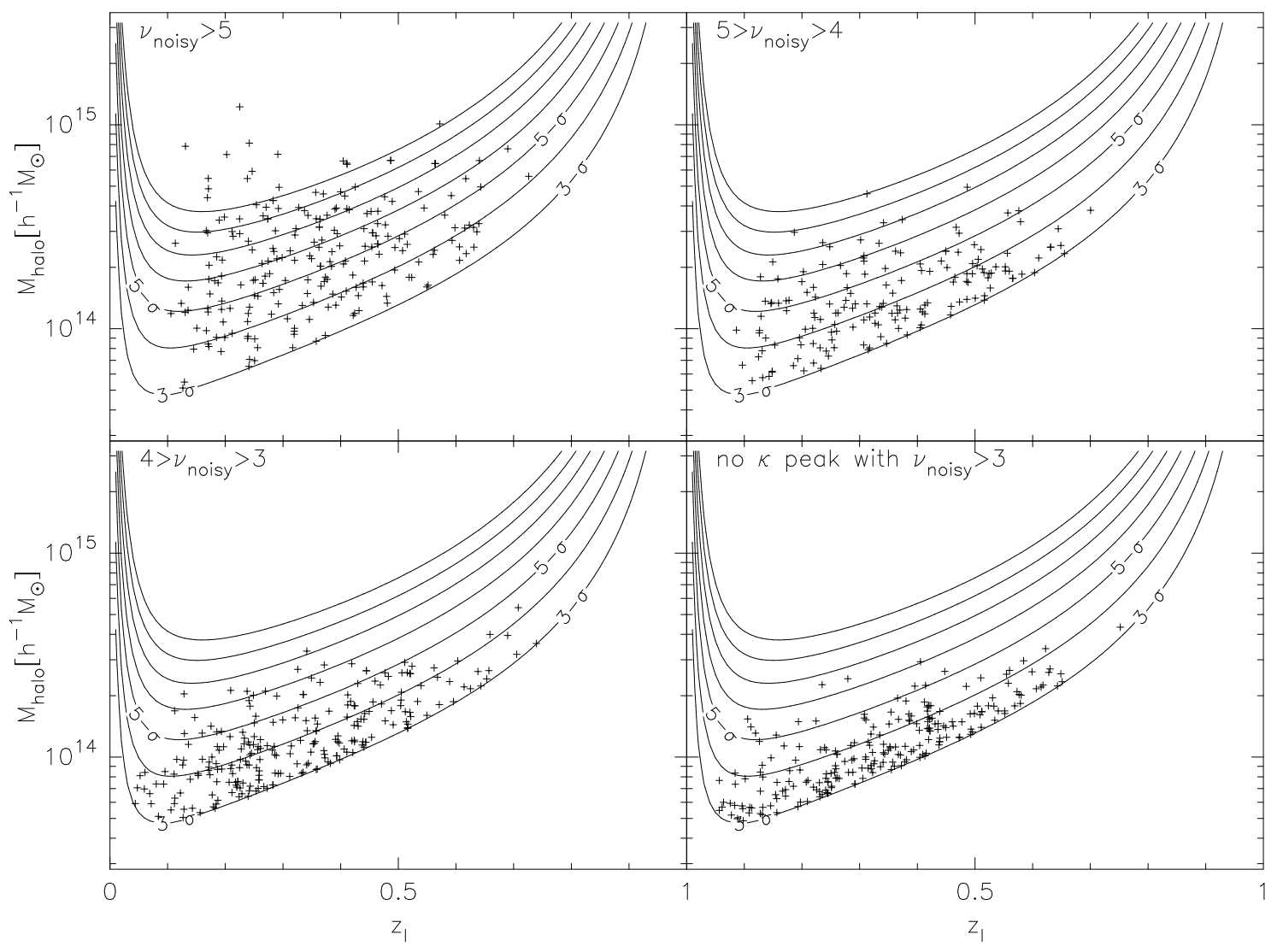

Figure 20. Same as Figure 17 but for halos matched (not matched, for lower-right panel) with peaks identified in noisy $\kappa$ maps.

The class-(ii) objects here correspond to the missing peaks, the peaks that have $\nu_{\text {lens }}>3$ but are absent in the corresponding noisy $\kappa$ map or have $\nu_{\text {noisy }}<3$. Except for one peak with $\nu_{\text {lens }}=5.1$, the missing peaks sit in the range of $\nu_{\text {lens }} \leqslant 4$. These peaks are likely to be erased or their heights are decreased by the addition of the negative noise. The one exception (the peak with $\nu_{\text {lens }}=$ 5.1 at the missing peak locus) is a local peak associated with a substructure of a massive halo which disappears in the noisy $\kappa$ map. We may, therefore, safely conclude that more than $80 \%$ of the peaks with $\nu_{\text {lens }} \geqslant 3$ in the noise-free map remain in the noisy $\kappa$ map.

Next, we consider the false peaks, the peaks that are identified in the noisy $\kappa$ map with $\nu_{\text {noisy }}>3$ but does not correspond to peaks in the noise-free $\kappa$ map or have $\nu_{\text {lens }}<3$, which are placed around $\nu_{\text {lens }} \sim 2$ in Figure 18 A large fraction of the false peaks, especially for $\nu_{\text {noisy }}<4$, are the false peaks generated by the noise (see 4 for details), and the small fraction (especially those with relatively high $\nu_{\text {noisy }}$ ) is associated with real peaks on which a large noise is accidentally added. Actually, there are $14 \mathrm{ob}-$ jects with $\nu_{\text {noisy }}>5$ in the false peaks locus. All of those have a corresponding peak in the noise-free $\kappa$ map with low peak height $1.5<\nu_{\text {lens }}<2.9$ on which a relatively large noise of $2 \sim 3.5 \sigma$ is accidentally added. Therefore, all peaks with $\nu_{\text {noisy }}>5$ identified in the noisy $\kappa$ map are associated with real peaks in the noise-free $\kappa$ map. On the other hand, most of the class-(iii) peaks with lower heights are false peaks generated by the noise. In fact, one can find the rapid increasing number of the false peaks toward low peak heights, especially for $\nu_{\text {noisy }}<4$, as shown in $\$ 4$ It immediately follows from this that in order to reduce the contamination rate, a high threshold value, $\nu_{\text {th }} \gtrsim 4$ at least, is required.

\subsection{Halos versus peaks in noisy $\kappa$ maps}

In this subsection, we examine correspondences between halos in the halo catalog and peaks in the $\kappa$ map including the noise due to the intrinsic ellipticities. Figure 19 shows the distribution of the class-(i-iii) objects in $\nu_{\mathrm{nfw}}-\nu_{\text {noisy }}$ plane plotted in the same manner as in Figure 14 The comparison with Figure 14 reveals that the noise not only increases the scatter in the $\nu_{\text {noisy }}-\nu_{\text {nfw }}$ relation but also causes the systematic bias in that the peak heights in the noisy map are statistically boosted, as seen in the previous subsection. Interestingly, this bias leads the completeness for the weak lensing halo search to be improved. The upper-right and lower-right panels in Figure 16 show the completeness and efficiency, defined in the same manner as eqs. (24) and (25), for the noisy maps. About 81 (63) percent of massive halos with $\nu_{\mathrm{nfw}}>5(>4)$ are identified as peaks with $\nu_{\text {noisy }} \geqslant 4$ in the noisy maps. This is one of the most important results of this paper, indicating that a high completeness of the weak lensing halo search could be attained in a realistic data.

There are 13 missing halos that have $\nu_{\mathrm{nfw}}>5$ but $\nu_{\text {noisy }}<3$ or have no corresponding peak in the noisy $\kappa$ map. We look into each case in the $\kappa$ map and find that 11 halos out of 13 are irregular systems such as un-relaxed systems having substructures or on-going merger. Therefore, the completeness of the weak lensing halo search for regular, well relaxed systems would be higher than the estimate plotted in Figure 16 The remaining 2 missing halos, which have $\nu_{\mathrm{nfw}}=5.3$ and 5.4, are halos which accidentally meet a large negative noise so that their $\kappa$ values are below $\nu_{\text {noisy }}=3$. The fraction of such halos over all halos with $\nu_{\mathrm{nfw}}>5$ is $0.8 \%$ (2 out of 250 halos), which is indeed very small. Hence, we can safely 


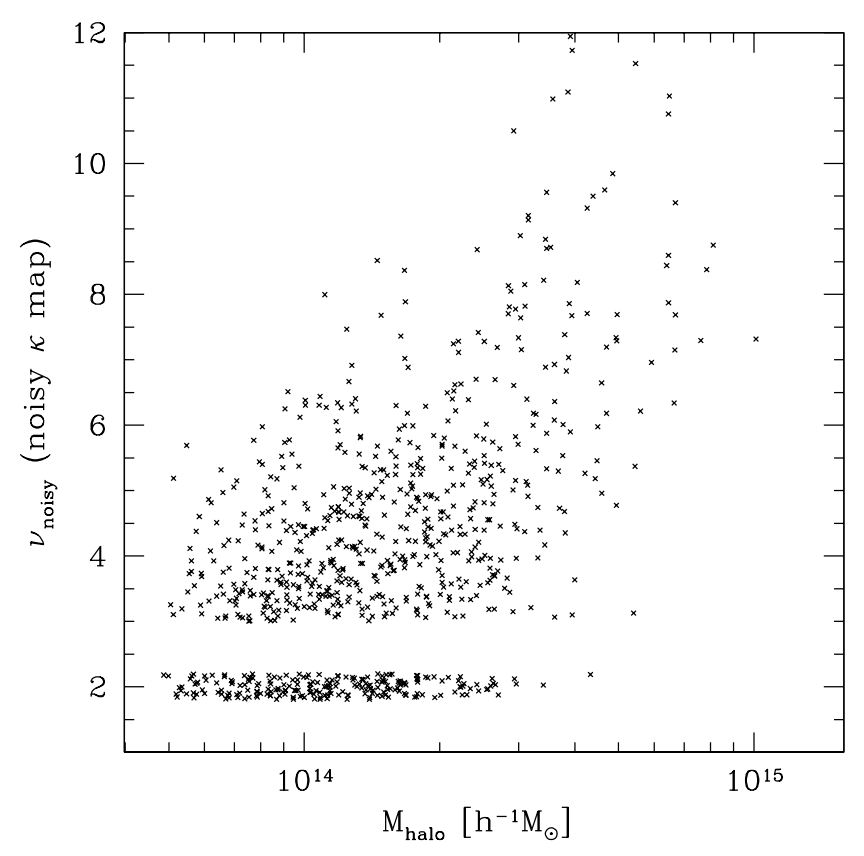

Figure 21. Same as Figure 19 but the halo mass is used instead of the expected peak height of $\nu_{\mathrm{nfw}}$. Note that only halos with $\nu_{\mathrm{nfw}}>3$ are considered.

conclude that almost all halos with $\nu_{\mathrm{nfw}}>5$ generate peaks with $\nu_{\text {noisy }}>3$ even in the presence of the noise.

Let us turn to the class-(iii) objects. Since the noise generates false peaks in the $\kappa$ map, the efficiency becomes worse than the noise-free case (see Figure 16. The fraction of contamination by the false peaks is larger for a lower peak height because the noise generates relatively lower peaks, mostly $\nu_{\text {noisy }}<4$. Therefore, if $\nu_{\text {th }}=3$ is employed as the detection threshold, only $28 \%$ of all peaks are associated with halos with $\nu_{\mathrm{nfw}}>3$. Note that since we only consider halos with $\nu_{\mathrm{nfw}}>3$, some fraction of missing peaks could be associated with halos with $\nu_{\mathrm{nfw}}<3$. On the other hand, the efficiencies for higher detection thresholds are reasonably high: for $\nu_{\mathrm{th}}=5\left(\nu_{\mathrm{th}}=4\right), 85 \%(56 \%)$ of all peaks with $\nu_{\text {noisy }}>\nu_{\mathrm{th}}$ are associated with halos with $\nu_{\mathrm{nfw}}>3$.

From Figure 16 it is clear that a better efficiency achieved by setting a higher detection threshold is a "trade-off" for a worse completeness. We also note that the peak counts varies sensitively with the threshold. For example, the counts $N(\nu>4)$ is about twice as large as $N(\nu>5)$ (see $\S 4)$. Hence, the detection threshold should be determined such that the efficiency and completeness are optimized for specific survey strategies. The results we have so far shown indicate that $\nu_{\text {noisy }} \simeq 4-5$ is reasonably optimal for currently typical weak-lensing surveys. To be more specific, the results from the simulated $\kappa$ maps in the $\Lambda$ CDM model indicate that, the mean number of halos with $\nu_{\mathrm{nfw}}>4$ is $N_{\text {halo }}\left(\nu_{\mathrm{nfw}}>4\right)=37$ per $10 \mathrm{deg}^{2}$, and on average $63 \%$ of such halos (23 halos out of 37) are detected with $\nu_{\text {noisy }}>4$. The completeness is better for higher $\nu_{\mathrm{nfw}}$ halos: for halos with $\nu_{\mathrm{nfw}}>5$, the mean number is $N_{\text {halo }}\left(\nu_{\text {nfw }}>5\right)=19$ per $10 \mathrm{deg}^{2}$, where $81 \%$ of the halos $(15$ halos out of 19) are detected with $\nu_{\text {noisy }}>4$, and 12 halos have even high lensing signal of $\nu_{\text {noisy }}>5$. Concerning the efficiency, if one sets the detection threshold to be $\nu_{\text {th }}=4$, the mean number of peaks is $N_{\text {peak }}\left(\nu_{\text {noisy }}>4\right)=62$ per $10 \mathrm{deg}^{2}$. Among the 62 peaks, 23 are due to real halos with $\nu_{\mathrm{nfw}}>4$, the 13 peaks are halos with $3<\nu_{\mathrm{nfw}}<4$ and the remaining 26 peaks are likely to

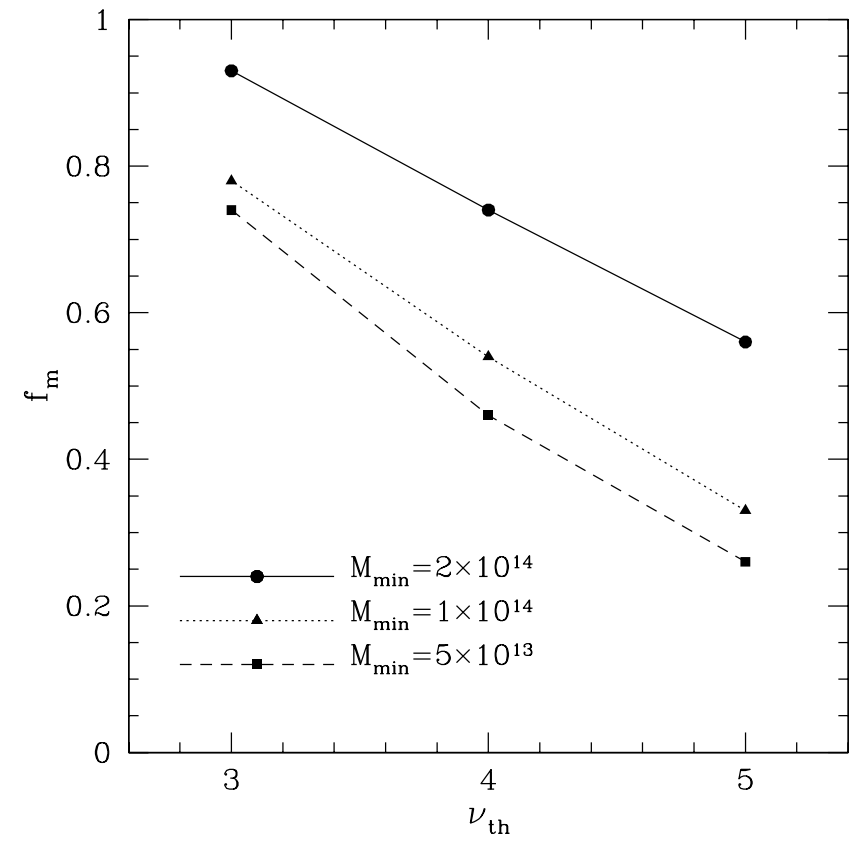

Figure 22. The number fraction of halos identified by peaks with height higher than $\nu_{\text {th }}$ relative to all the halos with mass greater than a given minimum mass, $M_{\min }, f_{m}\left(\nu_{\text {noisy }}>\nu_{\text {th }} \mid M_{\text {halo }}>M_{\min }\right)$ (see eq. (26). Three cases of $M_{\min }=5 \times 10^{13}, 1 \times 10^{14}$, and $2 \times 10^{14} h^{-1} M_{\odot}$ are plotted. Note that only halos with $\nu_{\mathrm{nfw}}>3$ are considered.

be either halos with $\nu_{\mathrm{nfw}}<3$ or false peaks due to the noise. On the other hand, if one adopts a higher detection threshold $\nu_{\text {th }}=5$, the mean number of peaks is reduced to $N_{\text {peak }}\left(\nu_{\text {noisy }}>5\right)=25$ per $10 \mathrm{deg}^{2}$, however the completeness is improved significantly: in fact it is found that the 19 (3) peaks out of the 25 are signals from real halos with $\nu_{\text {nfw }}>4\left(3<\nu_{\text {nfw }}<4\right)$.

Figure 20 shows the scatter plot for the class-(i) and class(ii) halos in the redshift-halo mass plane as in Figure 17 From comparison with Figure 17 one may find that more halos pass a certain detection threshold than in the noise-free map, because of the systematic bias caused by the noise. Another important point revealed in this figure is that the peak height in the noisy $\kappa$ map is a poor estimator of the halo mass. For example, masses of the halos detected with $4<\nu_{\text {noisy }}<5$ span over an order of magnitude. Even in a narrow redshift range such as $0.3<z<0.4$, there still exits a significant scatter in the halo mass. Note that we do not plot halos with $\nu_{\mathrm{nfw}}<3$, which may cause a larger scatter toward small mass ranges. It should be also noted that in the distribution of the missing halos (lower-right panel) no strong tendency with redshift is seen, indicating that the completeness would not vary significantly with redshift. This is largely due to our definition of the completeness, that is, we define the completeness so that the effect of the selection function, which very strongly depends on the redshift, is decoupled.

So far we have examined the relation between halos and peaks in a $\kappa$ map in terms of the peak height instead of the halo mass because the lensing observable is determined by combination of the halo mass, the redshift and the noise level, rather than by the halo mass alone. Nevertheless, it is interesting to see what mass range of halos produce the peaks in the noisy $\kappa$ maps, which is shown in Figure 21 as in Figure 19 Again only halos with $\nu_{\mathrm{nfw}}>3$ are considered. As can be seen, the peaks with a given height are caused by halos with a wide range of masses. We define the number 
fraction on the analogy of the completeness defined by eq. 24 but replacing the minimum peak height with the minimum halo mass:

$$
\begin{aligned}
f_{m}\left(\nu_{\text {noisy }}>\nu_{\text {th }} \mid M_{\text {halo }}>M_{\text {min }}\right)= \\
\frac{N_{\mathrm{i}}\left(\nu_{\text {noisy }}>\nu_{\text {th }} \mid M_{\text {halo }}>M_{\text {min }}\right)}{N_{\mathrm{i}+\mathrm{ii}}\left(M_{\text {halo }}>M_{\min }\right)},
\end{aligned}
$$

where $N_{\mathrm{i}+\text { ii }}\left(M_{\text {halo }}>M_{\min }\right)$ is the number of (class-(i) and (ii)) halos with mass greater than $M_{\text {min }}$, and $N_{\mathrm{i}}\left(\nu_{\text {noisy }}>\nu_{\text {th }} \mid M_{\text {halo }}>\right.$ $\left.M_{\min }\right)$ is the number of halos with mass greater than $M_{\min }$ having peak height greater than $\nu_{\mathrm{th}}$. Figure 22 shows this estimate as a function of $\nu_{\text {th }}$. It is apparent that $f_{m}$ declines toward higher detection threshold and for lower minimum mass. For detection threshold $\nu_{\text {th }}=4$, more than 70 percent of halos with $M_{\text {halo }}>$ $2 \times 10^{14} h^{-1} M_{\odot}$ are identified, while about half of halos with $M_{\text {halo }}>5 \times 10^{13} h^{-1} M_{\odot}$ is identified,

\section{SUMMARY AND DISCUSSION}

We have investigated various aspects of the weak lensing cluster surveys employing both analytic descriptions of dark matter halos and the mock data of weak lensing surveys generated from numerical simulations. For the latter, we combined weak lensing ray-tracing through the mass distribution and the dark matter halo catalogs. Our major findings are summarized as follows.

(1) In $\$ 2$ we examined the expected properties of weak lensing halos using the analytic descriptions of the dark matter halos, including the universal density profile (Navarro et al. 1996; 1997) and the Press-Schechter halo mass function (Press \& Schechter 1974: Sheth \& Tormen 1999). We found that the Gaussian smoothing with $\theta_{G} \simeq 1$ arcmin gives the largest expected weak lensing halo counts (which may however depend on the source redshift and noise properties). We computed the selection function of the weak lensing cluster survey and examined, in detail, the mass and redshift distribution of the weak lensing halos. It was shown that the detectability of halos depends not only on the mass but also strongly on the redshift.

(2) In 3.4 we compared the model prediction of the weak lensing halo counts developed by Kruse \& Schneider (2000) and Bartelmann et al. (2001) with the halo counts in the mock catalog, and found a good agreement. We also found a large scatter in the numbers of weak lensing halos within 4 square degree field, which is larger than the Poisson fluctuation. This can be explained by the strong clustering of massive halos.

(3) In 4 we tested the model prediction of the peak counts against the mock weak lensing data. It was found that a systematic bias is induced by the noise due to intrinsic galaxy ellipticities. This bias increases the peak counts, and hence the model prediction underestimates the counts. We developed a correction scheme in an empirical manner in Appendix $B$ and showed that the improved model reproduces the peak counts reasonably well.

(4) In 5.2 using the mock weak lensing data combined with the halo catalog, we examined the matching between halos and peaks identified in the noise-free $\kappa$ map. This was done to clarify influence of the individuality of halos and the projection effect on a weak lensing halo search. We showed that these effects cause not only a large scatter in the $\nu_{\text {lens }}-\nu_{\text {nfw }}$ relation but also a systematic bias. The mean and RMS of the differences $\nu_{\text {lens }}-\nu_{\mathrm{nfw}}$ are -0.24 and 1.2 , respectively. The level of scatter caused by the effects is comparable to that due to the noise. We argue that the negative mean value are due to a population of un-relaxed, less centrally concentrated halos and due to the fact that the more than half of the halos are elongated to the direction perpendicular to the line-of-sight. Also the projection of large under-dense regions may partially account for the scatter. It was also shown that the chance projection of massive halos in the same line-of-sight is very rare.

(5) In 5.3 we used the noise-free $\kappa$ map and the noisy $\kappa$ map to clarify how the peak distribution is affected by the noise and how false peaks are generated. We showed that the $\nu_{\text {lens }}-\nu_{\text {noisy }}$ distribution is biased toward large $\nu_{\text {noisy }}$ values. The mean and RMS of the differences, $\nu_{\text {noisy }}-\nu_{\text {lens }}$, among the matched peaks with $\nu_{\text {lens }}>4$ and $\nu_{\text {noisy }}>4$ are 0.31 and 0.90 , respectively. Thus the noise not only generates the scatter but also systematically 'boosts' the peak heights. We found that almost all the peaks identified in the noise-free $\kappa$ map with $\nu_{\text {lens }}>4$ are identified in the noisy $\kappa$ map, thus the noise rarely erases the high peaks. All the peaks with $\nu_{\text {noisy }}>5$ identified in the noisy $\kappa$ map are associated with real peaks in the noise-free $\kappa$ map, whereas most of the false peaks (due to the noise) have a relatively lower peak height $\nu_{\text {noisy }}<4$.

(6) In 5.4 we examined the correspondence between the halos and peaks identified in the noisy $\kappa$ map. In particular, we studied the efficiency and completeness of the weak lensing halo survey. We found that the detection threshold of $\nu_{t h} \simeq 4-5$ gives an optimal balance between the efficiency and completeness. It was shown that about 81 (62) percent of massive halos with $\nu_{\mathrm{nfw}}>5$ $(>4)$ are identified as high peaks with $\nu_{\mathrm{th}}=4$. This suggests that the completeness of the weak lensing cluster survey is reasonably high. Concerning the efficiency, we found that for $\nu_{\text {th }}=4(5), 58 \%$ (82\%) of all the peaks are real signals from halos with $\nu_{\mathrm{nfw}}>3$, while $37 \%(63 \%)$ are signals from halos with higher expected peak height of $\nu_{\mathrm{nfw}}>4$. Therefore, it is possible to attain relatively high efficiency and high completeness by selecting a moderately high detection threshold.

(7) In 5.4 we conclude that, for detection threshold $\nu_{\text {th }}=4$, about a half of halos with $M_{\text {halo }}>5 \times 10^{13} h^{-1} M_{\odot}$ and more than 70 percent of halos with $M_{\text {halo }}>2 \times 10^{14} h^{-1} M_{\odot}$ indeed produce the lensing signals with $\nu_{\text {noisy }} \geqslant 4$.

Weak lensing cluster search technique explored in this paper can be directly applied to data obtained by on-going/future wide field surveys. Extending the pilot 2.1 square degree survey (Miyazaki et al. 2002), a wide field weak lensing survey (Suprime33, PI: S. Miyazaki) is being carried out with the wide field prime focus camera on Subaru telescope, Suprime-Cam (Miyazaki et al. in preparation). The Suprime33 will cover 33 square degrees in total with the limiting magnitude $R=25.5$ (which provides $n_{g} \gtrsim 30$ and $\left\langle z_{s}\right\rangle \simeq 1$ ). For the survey exposure time, $1800 \mathrm{sec}$, and the field-of-view of the Suprime-Cam, 0.25 square degrees, the effective survey cost is 2.5 hours per 1 square degrees including the overhead. The expected number of clusters to be located by the Suprime33 is 70 (120) for the threshold $\nu_{\text {th }}=5$ $\left(\nu_{\text {th }}=4\right)$. Therefore weak lensing surveys exploiting a wide field camera on a large telescope offer a reasonably efficient way to locate massive clusters. The CFHT Legacy Survey ${ }^{4}$ will significantly enlarge the survey area. Its "wide" survey will observe 170 square degrees in total with the limiting magnitude $i^{\prime}=25.5$ (which provides $n_{g} \sim 30$ and $\left\langle z_{s}\right\rangle \simeq 1$ ). The expected number of clusters is $360(600)$ for $\nu_{\mathrm{th}}=5\left(\nu_{\mathrm{th}}=4\right)$, and thus it will provide invaluable data for studies on large-scale structure of the universe. Although we have primarily considered weak lensing surveys that are feasible with current ground-based telescopes, future wide field surveys based on a space telescope will enable to accurately measure the

\footnotetext{
${ }^{4}$ http://www.cfht.hawaii.edu/Science/CFHLS/
} 
shape of distant very small galaxy images, which significantly improves the ability of weak lensing cluster surveys and allows to detect lower mass and/or higher redshift clusters.

Finally, we note that it is in principle possible to enhance the $\mathrm{S} / \mathrm{N}$ of the detection by choosing a suitable smoothing function (White et al. 2002; Padmanabhan et al. 2003). Padmanabhan et al. (2003) proposed the function of $W(\theta)=\left(1+\theta / \theta_{c}\right)^{-2}$ with $\theta_{c} \sim 1$ arcmin, which is motivated by the asymptotic behavior of the outer part of the projected NFW profile. Such optimized filters may improve the $\mathrm{S} / \mathrm{N}$ if the matter distribution is indeed close to the NFW profile. On the other hand, its applicability to clusters whose mass distribution deviates from the NFW profile remains unclear. It needs to be explored how much improvement can be obtained both in efficiency and completeness. Optimizing the smoothing function clearly warrants further studies.

\section{ACKNOWLEDGMENTS}

We are grateful to Bhuvnesh Jain for many valuable discussions. We would like to thank Satoshi Miyazaki for useful discussions. We thank the anonymous referee for detailed and constructive comments on the earlier manuscript which improved various aspects of the paper. T.H. and N.Y. thank University of Pennsylvania for the warm hospitality during their visit where this work was initiated. T.H. and N.Y. acknowledge supports from Japan Society for Promotion of Science (JSPS) Research Fellowships. The $N$-body simulations used in this work were carried out by the Virgo Consortium at the computer center at Max-Planck-Institut, Garching (http://www.mpa-garching.mpg.de/NumCos). Numerical computations presented in this paper were partly carried out at ADAC (the Astronomical Data Analysis Center) of the National Astronomical Observatory, Japan.

\section{REFERENCES}

Bardeen J. M., Bond J. R., Kaiser N., Szalay A. S. 1986, ApJ, 304, 15

Bartelmann M., 1996, A\&A, 313, 443

Bartelmann M., King, L. J., Schneider P., 2001, A\&A, 378, 361

Bartelmann M., Schneider P., 1992, A\&A, 259, 413

Bartelmann M., Schneider P., 2001, Phys. Rep., 340, 291

Bond, J. R., Efstathiou, G., 1987, MNRAS, 226, 655

Bullock J. S., Kolatt T. S., Sigad Y., Somerville R. S., Kravtsov A. V., Klypin A. A., Primack J. R., Dekel A. 2001, MNRAS, 321, 559

Hamana, T., Mellier, Y. 2001, MNRAS, 169, 176

Hamana T., Yoshida N., Suto Y., 2002, ApJ, 568, 455

Henry, J. P. 2000, ApJ, 534, 565

Hockney, R. W., Eastwood, J. W., 1988, Computer Simulation Using Particles (Adam Hilger, Bristol)

Hoekstra H., 2001, A\&A, 370, 743

Jain, B., Seljak, U., White, S. D. M., 2000, ApJ, 530, 547

Jain, B., Van Waerbeke, L., 2000, ApJ, 530, L1

Jenkins A., Frenk C. S., White S. D. M., Colberg J. M., Cole S., Evrard A. E., Couchman H. M. P., Yoshida N., 2001, MNRAS, 321,372

Jing, Y. P., 2000, ApJ, 535, 30

Kaiser, N., Squires, G., 1993, ApJ, 404, 441

Kruse, G., Schneider, P., 2000, MNRAS, 318, 321
Macfarland, T., Couchman, H. M. P., Pearce, F. R., Pichlmeier, J., 1998, New Astronomy, 3, 687

Mellier Y., 1999, ARA\&A, 37, 127

Metzler C. A., White M., Loken C., 2001, ApJ, 547, 560

Miyazaki, S., et al., 2002, ApJ, 580, L97

Nakamura, T. T., \& Suto, Y. 1997, Prog. Theor. Phys., 97, 49

Navarro J., Frenk C., White S. D. M. 1996, ApJ, 462, 563

Navarro J., Frenk C., White S. D. M. 1997, ApJ, 490, 493

Padmanabhan N., Seljak U., Pen, U. L., 2003, New Astronomy, 8, 581

Press W. H., Schechter P., 1974, ApJ, 187, 425

Schneider, P., Ehlers, J., Falco, E. E., 1992, Gravitational Lenses, (Heidelberg: Springer Verlag)

Seljak, U., Zaldarriaga, M. 1996, ApJ, 469, 437

Sheth R. K., Tormen G., 1999, MNRAS, 308, 119

Takada M., Jain B., 2003, MNRAS, 340, 580

Reblinsky K., Bartelmann M., 1999, A\&A, 345, 1

Van Waerbeke, L., 2000, MNRAS, 313, 524

Vale, C., White, M., 2003, ApJ, 592, 699

White, M., Van Waerbeke, L., Mackey, J., 2002, ApJ, 575, 640

Wittman, D., Tyson, J. A., Margoniner, V. E., Cohen, J. G., Dell'Antonio, I. P., 2001, ApJ, 557, L89

Wright, C. O., Brainerd, T. G., 2000, ApJ, 534, 34

Yoshida, N., Sheth, R., Diaferio, A., 2001, MNRAS, 328, 669

\section{APPENDIX A: VISUAL APPEARANCES OF SYSTEMS WITH IRREGULAR SIGNALS}

Here we present some $\kappa$ maps, in which missing halos (the class(ii)) or false peaks (the class-(iii)) with high signals $(\nu>5)$ exists, for examples of irregular systems. Figures A1 A5 show the (noisefree) convergence maps smoothed with Gaussian window function with $\theta_{G}=1$ arcmin. Pluses denote the center-of-mass position of halos concerned and the circles show their virial radius. Crosses denote the position of peaks.

Figures A1 A3 show three examples of missing halos with the expected peak value $\nu_{\mathrm{nfw}}>5$. Such high $\nu_{\mathrm{nfw}}$ missing halos mostly have an un-relaxed appearance and are probably on-going merger systems. Figures A4 and A5 show two examples of false peaks with $\nu_{\text {lens }}>5$. Note that false peaks are rare in noise-free $\kappa$ maps. Most of false peaks with $\nu_{\text {lens }}>5$ are associated with substructures of massive halos. Occurrence of the chance projections is very rare, we found three cases with $\nu_{\text {lens }}>5$ in our 30 maps.

\section{APPENDIX B: SIMPLE CORRECTION SCHEME TO THE PEAK COUNTS}

As shown in 4 the theoretical model of the peak counts given by eq. (16) underestimates the counts obtained from the realistic noisy $\kappa$ map by about 20 percent. We here discuss sources of this change, and develop the correction scheme to the peak counts in an empirical manner. Here we focus on the high peaks of $\nu>5$.

The sources that modify the peak counts are:

(a) The biased relation between $\nu_{\mathrm{nfw}}-\nu_{\text {lens }}$. In 5.2 it is found that the mean of the differences $\nu_{\mathrm{nfw}}-\nu_{\text {lens }}$ over class-(i) objects is -0.24 . This means that the peak height computed assuming the NFW profile tends to overestimate the real peak height. The reason for this is discussed in 5.2 


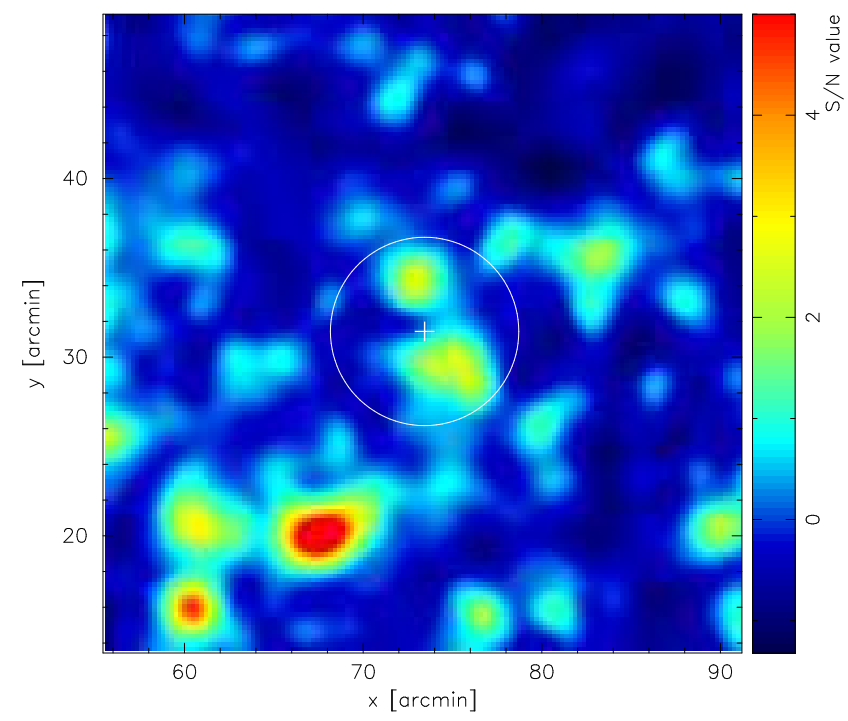

Figure A1. Missing halo, an example of a halo with extended matter distribution (possibly in the process of merger). The plus denotes the centerof-mass position of the halo, and the circle shows the virial radius. The map apparently shows very eccentric density distribution. The halo has $M_{\text {halo }}=3.4 \times 10^{14} h^{-1} M_{\odot}$ at $z=0.37$, and the shape parameters are $Q / Q_{\mathrm{NFW}}=2.1, R=1.4$, and $c / a=0.23$. The expected peak value is $\nu_{\mathrm{nfw}}=7.0$, but the $\nu$ value measured in the lensing map is less than 3 .



Figure A2. Missing halo, an example of low- $z$ un-relaxed system. The halo $\left(M_{\text {halo }}=2.0 \times 10^{14} h^{-1} M_{\odot}, z=0.13\right.$ and the shape parameters are $Q / Q_{\mathrm{NFW}}=1.2, R=0.36$, and $c / a=0.41$ ) seems to be not yet reached to a relaxed stage, but probably is a on-going merger system. The expected peak value is $\nu_{\mathrm{nfw}}=6.6$ but the actual $\nu$ value is less than 3 .

(b) Spurious peaks generated by the noise. It is found in the $\$ 5$ that spurious peaks yield about $10 \%$ fraction of the total number of high peaks $(\nu>5)$.

(c) The scatter in the relation between $\nu_{\text {noisy }}-\nu_{\text {lens }}$ due to the noise. As will be shown, this scatter increases the peak counts.

(d) The biased relation between $\nu_{\text {noisy }}-\nu_{\text {lens }}$. In 5.3 it is found that the noise produces not only the scatter but also the systematic bias, and that the mean of the differences $\nu_{\text {noisy }}-\nu_{\text {lens }}$ over class(i) objects is 0.31 . Therefore the peak heights in the noisy $\kappa$ map are

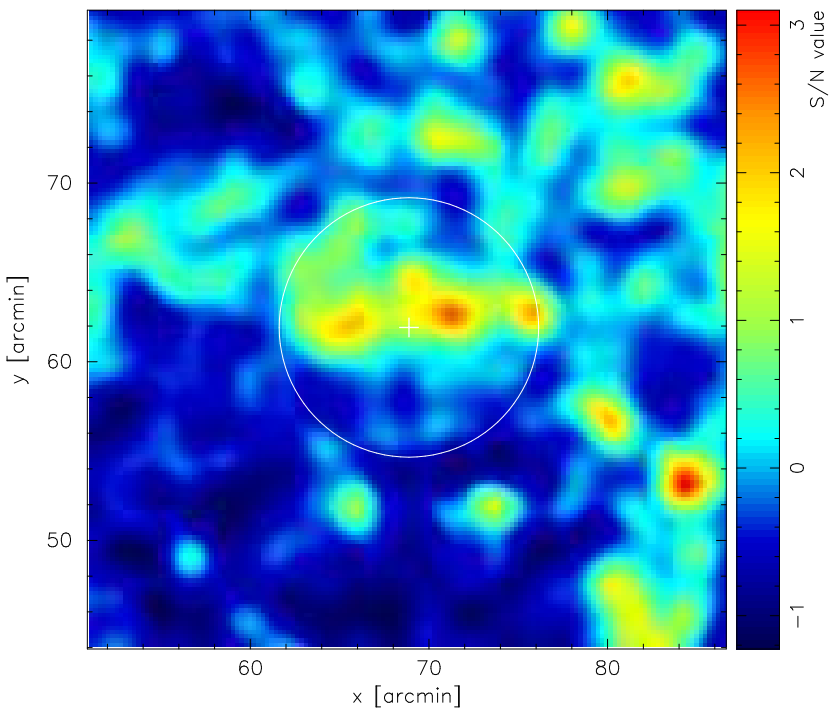

Figure A3. Missing halo, an example of an irregular system. The map apparently shows very eccentric density distribution. It is very likely that the halo $\left(M_{\text {halo }}=1.9 \times 10^{14} h^{-1} M_{\odot}, z=0.20\right.$ and the shape parameters are $Q / Q_{\mathrm{NFW}}=1.3, R=0.25$, and $c / a=0.36$.) is a on-going merger system. The expected peak value is $\nu_{\mathrm{nfw}}=6.1$ but the actual $\nu$ value is less than 3.

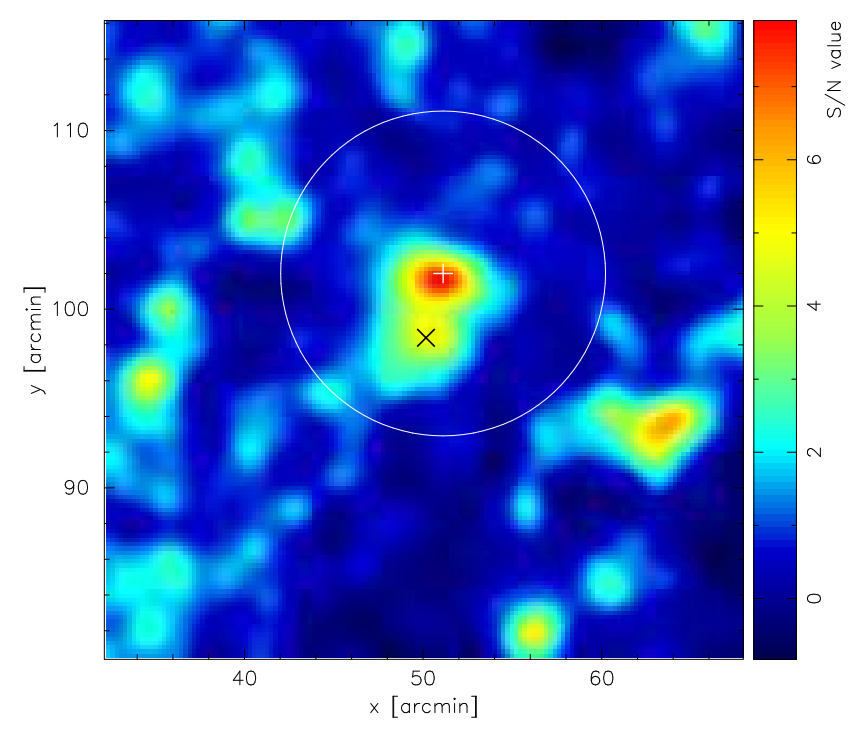

Figure A4. An example of a local peak associated with the substructure of a massive halo. The cross denotes the position of the peak with $\nu_{\text {lens }}=5.3$. This is the local peak associated with the substructure of the massive halo $\left(M_{\text {halo }}=3.5 \times 10^{14} h^{-1} M_{\odot}, z=0.20, \nu_{\mathrm{nfw}}=8.8\right.$ and the shape parameters are $Q / Q_{\mathrm{NFW}}=0.94, R=0.61$, and $\left.c / a=0.66\right)$. The plus denotes the center-of-mass position of the halo, and the circle shows the virial radius.

statistically boosted by the noise. The reason for this is discussed in $\$ 5.3$

First, we develop the correction scheme for the above point (c). To do this, we take a simplified way, employing the assumption that very high peaks are neither disappeared nor generated by the noise but their peak heights are altered by addition of the noise. In other words, we ignore, for the moment, spurious peaks due to the noise and the other sources. Under the above assumption, the peak 


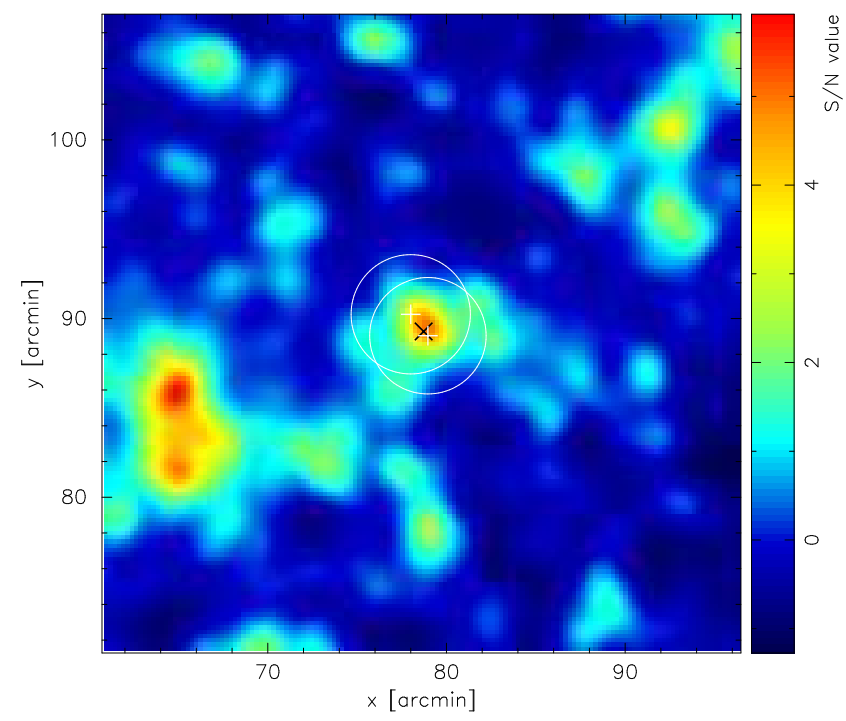

Figure A5. A very rare example of a chance projection of two moderately massive halos. The cross denotes the position of the peak with $\nu_{\text {lens }}=5.1$. This peak is not generated by a single halo but generated by the chance projection of two moderately massive halos: $\left(M_{\text {halo }}=7.5 \times 10^{13} h^{-1} M_{\odot}\right.$, $z=0.35)$ and $\left(M_{\text {halo }}=5.4 \times 10^{13} h^{-1} M_{\odot}, z=0.68\right)$. None of two halos alone can generate the lensing signal larger than $\nu>3$.

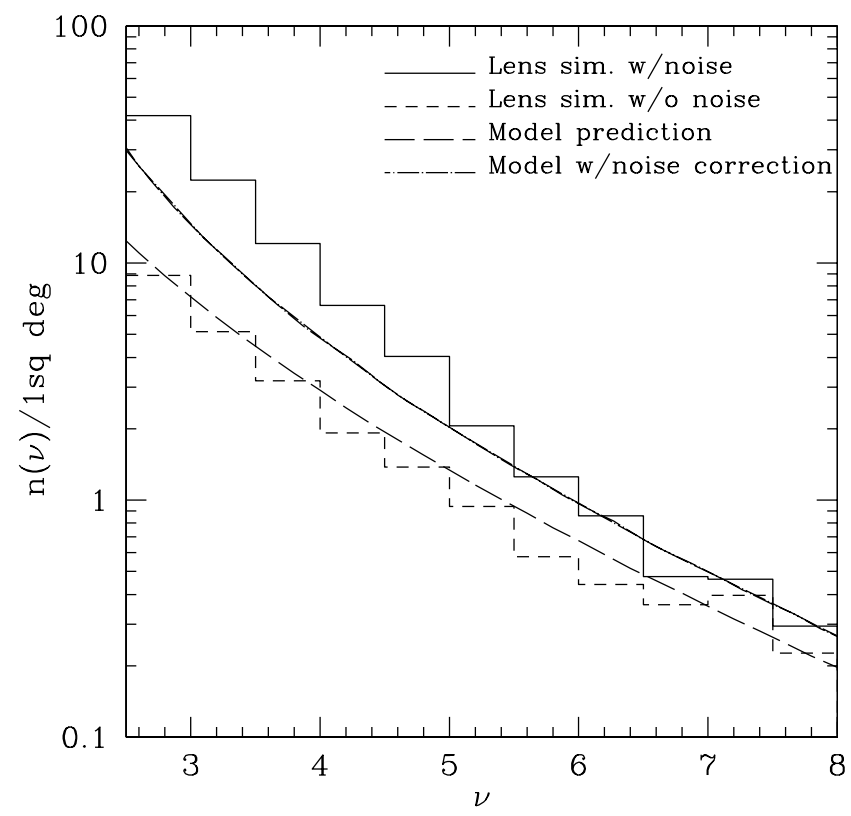

Figure B1. The number count of the lensing peaks per 1 squire degrees. The histograms are from the numerical experiments from the noisy $\kappa$ maps (solid), and the noise-free $\kappa$ maps (dashed). The long-dashed line shows the un-corrected model prediction computed from eq. 16, while the solid line shows the corrected model prediction (see text for detail).

counts in the noisy $\kappa$ map is given by the convolution of the noise free PDF (16) with the Gaussian noise PDF:

$n_{\text {noisy }}(\nu)=\int d \nu^{\prime} \frac{1}{\sqrt{2 \pi}} \exp \left(-\frac{\nu^{\prime 2}}{2}\right) n_{\text {peak }}\left(\nu-\nu^{\prime}\right)$.

We further assume that within a small range of $\nu$ the peak counts in the original lensing map can be approximated by the exponential form $n_{\text {peak }}(\nu)=n_{*} \exp (p \nu)$, with a constant exponential index of $p$. As can be seen in Figure B1 this would be a reasonable approximation over a range of $3 \lesssim \nu \lesssim 8$. This approximation allows us to compute the integration in eq. B1 analytically:

$$
\begin{aligned}
n_{\text {noisy }}(\nu) & \simeq \int d \nu^{\prime} \frac{1}{\sqrt{2 \pi}} \exp \left(-\frac{\nu^{\prime 2}}{2}\right) n_{*} \exp \left(p\left(\nu-\nu^{\prime}\right)\right) \\
& =\exp \left(p^{2} / 2\right) n_{\text {peak }}(\nu) .
\end{aligned}
$$

Thus the scatter due to the noise does increase the counts by a factor of $\exp \left(p^{2} / 2\right)$.

Turn next to the point (a) and (d) in the above list. From the biases in the relations $\nu_{\mathrm{nfw}}-\nu_{\text {lens }}$ and $\nu_{\text {lens }}-\nu_{\text {noisy }}$ found in the mock data, we derive an approximate mean relation of $\nu_{\text {noisy }}-$ $\nu_{\mathrm{nfw}}=0.07$. We take this bias by simply shifting the counts as $n_{\text {noisy }}(\nu) \rightarrow n_{\text {noisy }}(\nu+0.07)$.

Finally, the point (b) is corrected by simply increasing the peak counts by 10 percent.

In Figure B1 the corrected model prediction is plotted and is compared with the results from numerical experiments. To compute the corrected model prediction, $n_{\text {noisy }}(\nu)$, we first computed the uncorrected counts $n_{\text {peak }}(\nu)$ at a small interval of $\delta \nu$ using eq. 16. Then the local exponential slope of the counts is evaluated by a finite differential scheme $p\left(\nu_{i}\right)=\left[\log n\left(\nu_{i+1}\right)-\right.$ $\left.\log n\left(\nu_{i}\right)\right] / \delta \nu$. We compute $n_{\text {noisy }}(\nu)$ using the eq. B2] with this $p\left(\nu_{i}\right)$. Then, the correction $n_{\text {noisy }}(\nu) \rightarrow n_{\text {noisy }}(\nu+0.07)$ was made for $p\left(\nu_{i}\right)$ at every $\nu_{i}$ to correct the points (a) and (d). Finally the counts is increased by 10 percent to correct the point (b). The corrected prediction agrees with the noisy counts reasonably well at least $\nu>5$, where the above approximations are valid. On lower peak heights $\nu<4$, the prediction is systematically smaller than the measurement, where false peaks due to the noise have larger contribution. Note that the amplitude of the effects (b) and (d) may depend on the noise properties and on the choice of the smoothing scheme.

It is clearly seen in the Figure B1] that the corrected peak counts has a slope very similar to the un-corrected counts. Therefore, one may make a simple correction to the model prediction obtained from eq. 16 by multiplying the factor 1.2 , which gives an alternative approximate way to compute the corrected peak counts with a reasonable accuracy.

\section{APPENDIX C: RELATIONS BETWEEN THE HALO SHAPE AND $\kappa$ AMPLITUDE}

Here we examine the effect of the individuality in the halo mass distribution on the amplitude of the $\kappa$ peak. Figures C1 and C2 show how the ratio $\nu_{\text {lens }} / \nu_{\mathrm{nfw}}$ varies with the halo shape parameters $Q$ and $R$ (see equations (20) and 23) for the definitions), where we consider the class-(i) halos with $\nu_{\text {nfw }}>4$ and $\nu_{\text {lens }}>4$ only. The filled circles show the average over halos sitting in the range denoted by the $x$-direction bar, where each interval is defined so that it contains 35 halos, and the error bar in $y$-direction denotes the RMS among them.

The correlations between the shape parameters and amplitude of the lensing signal are observed in both the figures, though the statistical significance is not very high. The anti-correlation in the $\nu_{\text {lens }}-Q$ is because more concentrated mass distribution $(Q<1)$ compared to the average NFW profile provides greater lensing signal. The lensing signal is also amplified if mass distribution within a halo is elongated along the line-of-sight $(R>1)$. However, we 


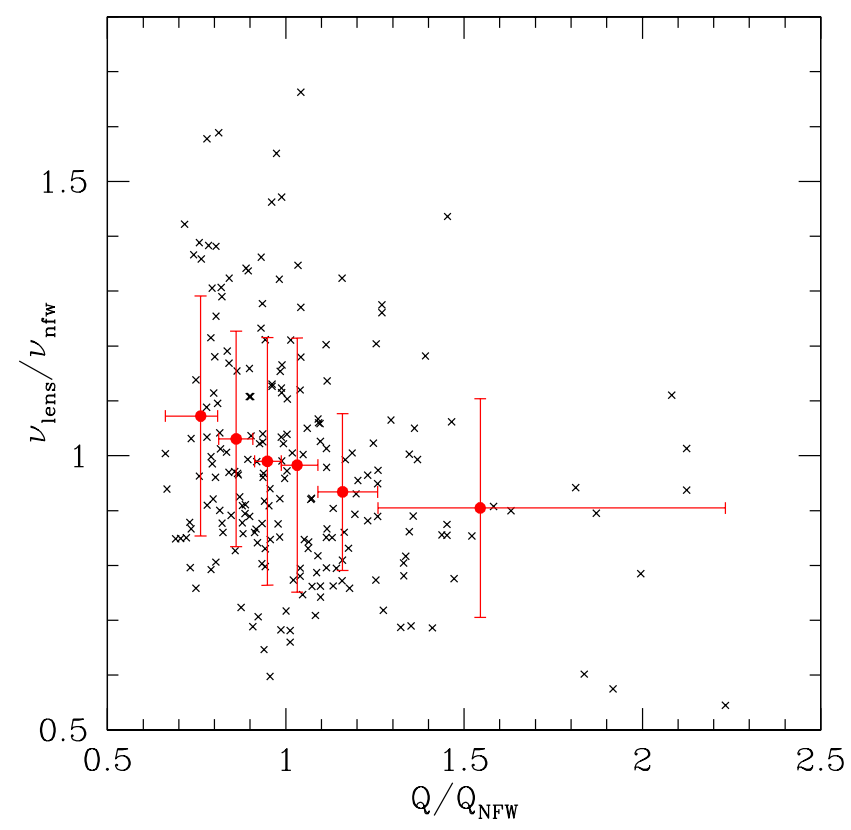

Figure C1. Shown is the scatter plot of the distribution of the halos in $Q / Q_{\mathrm{NFW}}-\nu_{\text {lens }} / \nu_{\mathrm{nfw}}$ plane. The class-(i) halos having $\nu_{\mathrm{nfw}}>4$ and $\nu_{\text {lens }}>4$ only are plotted. The filled circles show the average over 35 halos within bins whose ranges are denoted by the $x$-error bars, while the $y$-error bars denote RMS among the 35 halos.

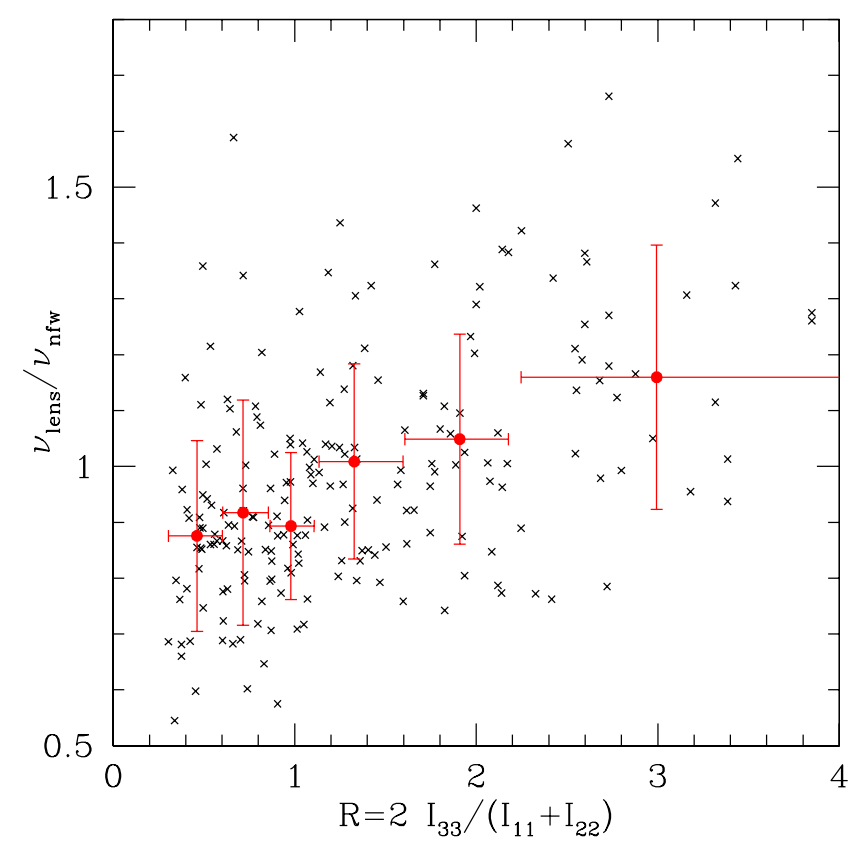

Figure C2. Same as Figure C1] but for $R-\nu_{\text {lens }} / \nu_{\text {nfw }}$ plane.

draw attention to the rather large scatters in these relations that may arise from the following two sources. First, the shape parameters we employ characterize rather global mass distribution within a halo and cannot describe the detailed structures. Second, probably more important one is the projection effect due to different structures along the same line-of-sight of the halo. 